\title{
Tracking Signal Subspace Invariance for Blind Separation and Classification of Nonorthogonal Sources in Correlated Noise
}

\author{
Karim G. Oweiss ${ }^{1}$ and David J. Anderson ${ }^{2}$ \\ ${ }^{1}$ Electrical \& Computer Engineering Department, Michigan State University, East Lansing, MI 48824-1226, USA \\ ${ }^{2}$ Electrical Engineering \& Computer Science Department, University of Michigan, Ann Arbor, MI 48109-2122, USA
}

Received 1 October 2005; Revised 11 April 2006; Accepted 27 May 2006

Recommended by George Moustakides

\begin{abstract}
We investigate a new approach for the problem of source separation in correlated multichannel signal and noise environments. The framework targets the specific case when nonstationary correlated signal sources contaminated by additive correlated noise impinge on an array of sensors. Existing techniques targeting this problem usually assume signal sources to be independent, and the contaminating noise to be spatially and temporally white, thus enabling orthogonal signal and noise subspaces to be separated using conventional eigendecomposition. In our context, we propose a solution to the problem when the sources are nonorthogonal, and the noise is correlated with an unknown temporal and spatial covariance. The approach is based on projecting the observations onto a nested set of multiresolution spaces prior to eigendecomposition. An inherent invariance property of the signal subspace is observed in a subset of the multiresolution spaces that depends on the degree of approximation expressed by the orthogonal basis. This feature, among others revealed by the algorithm, is eventually used to separate the signal sources in the context of "best basis" selection. The technique shows robustness to source nonstationarities as well as anisotropic properties of the unknown signal propagation medium under no constraints on the array design, and with minimal assumptions about the underlying signal and noise processes. We illustrate the high performance of the technique on simulated and experimental multichannel neurophysiological data measurements.
\end{abstract}

Copyright (C) 2007 K. G. Oweiss and D. J. Anderson. This is an open access article distributed under the Creative Commons Attribution License, which permits unrestricted use, distribution, and reproduction in any medium, provided the original work is properly cited.

\section{INTRODUCTION}

Multichannel signal processing aims at fusing data collected at several sensors in order to carry out an estimation task of signal sources. Generally speaking, the parameters to be estimated reveal important information characterizing the sources from which the data is observed. The aim of array signal processing is to extract these parameters with the minimal degree of uncertainty to enable detection and classification of these sources to take place. Many array signal processing algorithms rely on eigenstructure subspace methods performed either in the time domain, in the frequency domain, or in the composite time-frequency domain [1-3]. Regardless of which domain is used, eigenstructure based algorithms offer an optimal solution to many array processing applications provided that the model assumptions about the underlying signal and noise processes are appropriate (e.g., independent source signals, uncorrelated signals and noise, spatially and temporally white noise processes, etc.) [4-7].
For some applications, many of these assumptions cannot be intrinsically made, such that when the sources have correlated waveform shapes and the noise is correlated among sensors, or when the propagating medium is anisotropic. Many approaches have been suggested in the literature to mitigate the effects of unknown spatially correlated noise fields to enable better source separation of the array mixtures and showed various degrees of success (see [6-8] and the references therein). Nevertheless, the particular case where signal sources are nonorthogonal and may inherently possess considerable correlation with the contaminating noise has not received considerable attention. This situation may occur, for example, when the noise is the result of the presence of a large number of weak sources that generate signal waveforms identical to those of the desired ones. Recording of neuronal ensembles in the brain with microelectrode arrays is a classical example where such situation is frequently encountered $[9,10]$. 
The objective of this paper is to develop a new technique for separating and potentially classifying a number of correlated sources impinging on an array of sensors in the presence of strong correlated noise. Although we focus specifically on neural signals recorded by microelectrode arrays in the nervous system as the primary application, the technique is applicable to a wide variety of applications where similar signal and noise characteristics are encountered. The paper targets the source separation problem in detail, while the classification task using the features obtained is detailed elsewhere [11]. In that respect, we make the following assumptions about the problem at hand.

(1) The observations are an instantaneous mixture of wide-band signals.

(2) Sources are not in the far field, are nonorthogonal with signals that are transient-like, and may be fully or partially coherent across the array.

(3) The number of sources within the analysis interval is unknown.

(4) The noise is a mixture of two components:

(a) zero mean independent, identically distributed (iid) Gaussian white noise (e.g., thermal and electronic noise),

(b) correlated noise component with unknown temporal and spatial covariance resulting from numerous interfering weak sources.

The technique proposed exploits mainly spatial diversity in the signals observed under the assumptions stated above [12]. It does not attempt to exploit delay spread or frequency spread [13]. In that regard, we focus on the blind separation of the sources without trying to identify the channel. Though our model is the classical linear array model typically used in array processing literature, it does not assume a linear time invariant (LTI) finite impulse response (FIR) system to model the channel, as is the case in typical multipleinput multiple-output (MIMO) systems [13, 14]. Because of the existence of the sources in the proximity of the array, and the fact that the signal sources cannot be treated as point sources $^{1}$ as we will demonstrate later, classical direction of arrival (DOA) techniques are generally inapplicable.

The paper is organized as follows: Section 2 describes relevant array processing theory starting from the signal model in the absence of noise and in the presence of noise. Section 3 describes the advantages gained by orthogonal transformation prior to eigendecomposition. The formulation of the algorithm is detailed by analyzing the array model in the multiresolution domain. In Section 4, we demonstrate the performance of the algorithm using simulated and experimental data.

To clarify the notation, we will adhere to the somewhat standard notation convention. Uppercase, boldface characters will generally refer to random matrices, while uppercase, boldface nonitalic characters will generally refer to deter-

\footnotetext{
${ }^{1}$ In neurophysiological recording, every element of the signal source (neuron) is capable of generating a signal and therefore the signal source cannot be regarded as a point source [15].
}

ministic matrices (e.g., linear transformations). Lowercased boldfaced characters will generally refer to column vectors. Eigenvalues of square Hermitian matrices are assumed to be ordered in decreasing magnitude, as are the singular values of nonsquare matrices. The notation $(\cdot)_{j}$ will generally refer to a quantity estimated in the $j$ th frequency subband, except for correlation matrices, where the notation $(\cdot)_{Q}^{j}$ will be used to define the correlation of the $\mathbf{Q}$ data matrix estimated in the $j$ th frequency subband.

\section{MATHEMATICAL PRELIMINARIES}

Consider a model of $P$ signals impinging on an array of $M$ sensors expressed in terms of the $M \times 1$ signal vector over an observation interval of length $N$ :

$$
\mathbf{x}(n)=\operatorname{As}(n), \quad n=0, \ldots, N-1,
$$

where $\mathbf{A} \in \mathbb{R}^{M \times P}$ denotes the mixing matrix that expresses the array response to the $n$th snapshot of $P$ sources $\mathbf{s}(n)=$ $\left[\begin{array}{llll}s_{1}(n) & s_{2}(n) & \cdots & s_{p}(n)\end{array}\right]^{T}$, where $P \leq M$. Over the observation interval, each source $\boldsymbol{s}_{p}$ is assumed Gaussian distributed with zero mean and variance $\sigma_{s_{p}}^{2}, p=1, \ldots, P$. The model can be more conveniently expressed in matrix form as

$$
\mathbf{X}=\left[\begin{array}{llll}
\mathbf{x}(0) & \mathbf{x}(1) & \cdots & \mathbf{x}(N-1)
\end{array}\right]=\mathbf{A S} .
$$

This model is widely recognized in the array processing community when it is required to estimate the unknown source matrix $\mathbf{S}$ or their DOAs from an estimate of A. Alternatively, it is also used in MIMO systems in which a known source matrix $\mathbf{S}$ (training signals) is used to probe the transmission channel in order to estimate the unknown channel matrix. In our context, it is assumed that neither $\mathbf{A}$ nor $\mathbf{S}$ is known. This situation may occur, for example, in blind source separation problems where it is necessary to extract as many signals as possible from the observed data. The mixing matrix in this case models three elements: (1) the spatial extent of the source, (2) the transmission channel that characterizes the unknown signal propagation medium, and (3) the sensor point spread function [16].

Characterizing the unknown sources has been widely exploited using second-order statistics of the data matrix. First, we briefly review some known concepts using vector space theory. In model (2), the column space of the signal matrix $\mathbf{X}$ is spanned by all the linearly independent columns of $\mathbf{A}$, while the row space of $\mathbf{X}$ is spanned by the rows of $\mathbf{S}$. Using second-order statistics, the signal subspace, denoted $\{\mathbf{A}\}$, can be identified using singular value decomposition (SVD) as

$$
\mathbf{X}=\mathbf{U}_{X} \mathbf{D}_{X} \mathbf{V}_{X}^{T}
$$

When the sources are uncorrelated with unequal energy, then $\mathbf{R}_{S}=E\left[\mathbf{S S}^{T}\right]=\operatorname{diag}\left[\sigma_{\mathbf{S}_{1}}^{2}, \sigma_{\mathrm{S}_{2}}^{2}, \ldots, \sigma_{\mathbf{S}_{p}}^{2}\right]$. The largest $P$ eigenvalues of $\mathbf{R}_{X}=E\left[\mathbf{X X}^{T}\right]$ are nonzero and correspond to eigenvectors $\mathbf{U}_{S}=\left[\mathbf{u}_{1}, \mathbf{u}_{2}, \ldots, \mathbf{u}_{P}\right] \in \mathbb{R}^{M \times P}$ that span the subspace $\{\mathbf{A}\}$ spanned by the columns of $\mathbf{A}$. The remaining $M-P$ eigenvalues are zero with probability one, and the remaining eigenvectors $\left[\mathbf{u}_{P+1}, \mathbf{u}_{P+2}, \ldots, \mathbf{u}_{M}\right]$ span the null space 
of $\mathbf{A}$. This analysis is guaranteed to separate the sources from knowledge of $\mathbf{A}$, or a least squares (LS) estimate of $\mathbf{A}[4]$.

When the source signals are nonorthogonal, that is, $\left\langle\mathbf{s}_{i}, \mathbf{s}_{j}\right\rangle \neq 0$, where $\langle\cdot\rangle$ denotes a dot product, $\mathbf{R}_{S}$ has an $(i, j)$ th entry given by

$$
R_{S}(i, j)=\rho_{i j} \sigma_{s_{i}} \sigma_{s_{j}}=\sum_{p=1}^{P} \lambda_{p} \mathbf{u}_{p}[i] \mathbf{u}_{p}^{T}[j],
$$

where $\rho_{i j}$ expresses the unknown correlation between the $i$ th and $j$ th sources. Therefore, each eigenvalue $\lambda_{p}$ corresponds to the mixture of sources that have nonzero projection along the direction of eigenvector $\mathbf{u}_{p}$. Therefore, the strength of the $i$ th mode of the signal covariance can be expressed as

$$
\lambda_{i}=\sigma_{\mathbf{s}_{i}} \sum_{i=1}^{P} \rho_{i j} \sigma_{\mathbf{s}_{j}}, \quad i=1, \ldots, P .
$$

This results in an ambiguity in identifying the signal subspace. This occurs because each eigenvector spans a direction determined by the correlated component of the sources and not that of each individual source.

\section{ORTHOGONAL TRANSFORMATION}

\subsection{Noise-free model}

Our approach for solving this complex problem relies on exploiting an alternative solution to signal subspace determination. Recall from (4) that the signal subspace is a $P$ dimensional space that can be determined from the span of the columns of A. Alternatively, it can be determined from the $P$ rows of $\mathbf{S}$ if signal correlation is minimized by appropriate signal subspace rotation. If the rotation does not alter the span of the columns of $\mathbf{A}$, then it can be used to separate the correlated sources. This can be seen if the mixing matrix is decomposed as $\mathbf{A}=\mathbf{Q H}^{\mathrm{T}}$ [17]. The $M \times P$ matrix $\mathbf{Q}$ corresponds to a whitening matrix that can be determined from the data if training sequences are available. On the other hand, $\mathbf{H}$ is a $P \times P$ unitary rotation matrix on the space $\mathbb{R}^{P \times 1}$. In [17], a semiblind MIMO approach was suggested to determine $\mathbf{Q}$ and $\mathbf{H}$ from the pilot data (training sequence). However in the current problem, we stress the notion that the purpose is to blindly separate and classify $P$ unknown sources, and not to estimate the channel. Even if samples of the source signals are available for training after an initial signal extraction phase for example, they will not fulfill the orthogonality condition typically required in pilot signals. Because A can be expressed using SVD as $\mathbf{A}=\mathbf{E} \boldsymbol{\Sigma} \boldsymbol{\Gamma}^{T}$, then a suggested choice [17] for $\mathbf{Q}$ would be $\mathbf{Q}=\mathbf{E} \boldsymbol{\Sigma}$, while $\mathbf{H}=\boldsymbol{\Gamma}$. However, this factorization assumes that $\mathbf{A}$ is known. Note that the $M \times P$ matrix of eigenvectors $\mathbf{U}_{S}$ can be utilized as an alternative to finding $\mathbf{Q}$ from unavailable training data. However, there are two conditions that have to be satisfied in order to utilize $\mathbf{U}_{S}$ : (1) the signal sources have to be orthogonal with a sufficiently long data stream to avoid biasing the estimate of $\mathbf{Q}$, and (2) the number of sources $P$ to be separated is known to determine the number of columns of $\mathbf{U}_{S}$.
Clearly both conditions are inapplicable given the assumptions we stated above.

Our alternative approach is to approximately "null" the effect of the rotation matrix $\mathbf{H}$ on the source matrix $\mathbf{S}$. This can be achieved using a wide range of orthogonal transformation. The idea is to find a particular orthogonal transformation to undo the rotation caused by $\mathbf{H}$, or equivalently minimize signal correlation. For reasons that will become clear in the sequel, we opted to use an orthogonal basis set that projects the observation matrix onto a set of nested multiresolution spaces. This can be efficiently achieved using a discrete wavelet transformation (DWT) or its overcomplete version, the discrete wavelet packet transform (DWPT). The advantage of using the DWPT is the considerable sparseness it introduces in the transform domain. Besides, the DWPT orthogonal transformation is known to universally approximate a wide variety of unknown signals. Taken together, both properties will allow source separation to take place without having to estimate the matrix $\mathbf{H}$.

Let us denote by $\boldsymbol{W}^{(j)}$ an $N \times N$ DWPT orthogonal transformation operator at resolution $j$, where $j=0,1, \ldots, J$. Let us operate on the data matrix in (2), so we obtain

$$
\mathbf{X}_{j}=\mathbf{A S} \boldsymbol{W}^{(j)}=\mathbf{A S}_{j}, \quad j=0,1, \ldots, J
$$

where $\mathbf{S}_{j}$ denotes the source matrix projected onto the space $\Omega_{j}$ of all piecewise smooth functions in $L_{2}(\mathbb{R})$. These are spanned by the integer-translated and dilated copies $\phi_{j, k} \stackrel{\text { def }}{=}$ $2^{j / 2} \phi\left(2^{j} \cdot-k\right)$ of a scaling function $\phi$ that has compact support [18]. In practice, (6) is obtained by performing an undecimated DWPT projection on each row of $\mathbf{X}$ separately and stacking the results in the $M \times N$ matrix $\mathbf{X}_{j}$. Spectral factorization of (6) using SVD yields

$$
\mathbf{X}_{j}=\mathbf{U}_{X}^{j} \mathbf{D}_{X}^{j} \mathbf{V}_{X}^{j^{T}}=\sum_{i=1}^{M} \lambda_{i}^{j} \mathbf{u}_{i}^{j} \mathbf{v}_{i}^{j^{T}} .
$$

The columns of the eigenvector matrix $\mathbf{V}_{X}^{j}$ span the row space of $\mathbf{X}_{j}$, that is, the space spanned by the transformed signals $\mathbf{s}_{p}^{j}, p=1, \ldots, P$, which are now sparse. This means that $\mathbf{s}_{p}^{j}$ will have a few entries that are nonzero. The sparsity introduced by the DWPT operator enables us to infer a relationship between the row space of $\mathbf{X}_{j}$ and that of $\mathbf{X}$ using the whitening-rotation factorization of $\mathbf{A}$ discussed above. Specifically, if $\boldsymbol{W}^{(j)}$ spans the null space of the product $\mathbf{H}^{\mathrm{T}} \mathbf{S}$, the corresponding rows of $\mathbf{H}^{\mathrm{T}} \mathbf{S}_{\mathbf{j}}$ will be zero. Conversely, if $W^{(j)}$ spans the range space of $\mathbf{H}^{\mathrm{T}} \mathbf{S}$, then the corresponding rows of $\mathbf{H}^{\mathrm{T}} \mathbf{S}_{\mathbf{j}}$ will be nonzero. Furthermore, they will belong to the subspace spanned by the columns of the whitening matrix $\mathbf{Q}$, or equivalently $\mathbf{U}_{S}$.

Given the spectral factorization of $\mathbf{X}_{j}$ in (7), a necessary (but not sufficient) condition for a column of $\mathbf{V}_{X}^{j}$ to span the row space of $\mathbf{X}_{j}$ is the existence of at least one row of $\mathbf{H}^{\mathrm{T}} S_{\mathbf{j}}$ that is nonzero with probability one. If such a row exist, then a corresponding independent column in $\mathbf{U}_{X}^{j}$ will exist. This argument elucidates that any perturbation in the number of linearly independent columns in $\mathbf{V}_{X}^{j}$, which is directly 
associated with the number of distinct eigenvalues along the diagonal entries in $\mathbf{D}_{X}^{j}$, will directly impact the corresponding independent columns of $\mathbf{U}_{X}^{j}$. This can be seen from (7) using the outer product form.

To be more specific, let us denote by $\Delta\{J\}$ the full dictionary of basis obtained from a DWPT decomposition up to $L$ decomposition levels ${ }^{2}$ ( $J$ subbands). Among all the $J$ bases obtained, a subset of basis is selected from the dictionary $\Delta\{J\}$ for which $\mathbf{W}^{(j)}$ spans the range space of $\mathbf{H}^{\mathrm{T}} \mathbf{S}$. This subset is interpreted as the collection of wavelet basis that best represent the sources in the range space of $\mathbf{H}^{\mathrm{T}} \mathrm{S}$. Let us assume that $\mathbf{S}$ contains a single source, that is, $P=1$. Let us denote the subset of basis by $\mathfrak{J}_{1}$, and the cardinality of the set $\mathfrak{J}_{1}$ will be denoted $J_{1}$. This implies that there is only $J_{1}$ basis in the DWPT expansion for which $\mathbf{h}_{1}^{T} \mathbf{s}_{1}^{j}, j \in \mathfrak{J}_{1}$, is nonzero. Therefore, the signal subspace spanned by the columns of $\mathbf{U}_{X}^{j}$, denoted $\{\mathbf{A}\}_{j}$, will be restricted to those basis that belong to $\mathfrak{J}_{1}$ as evident from (7). We denote the signal subspace dimension in subband $j$ by $P_{j}$, where it is straightforward to show that $P_{j}$ is always upper bounded by $P[19]$.

Since $\mathcal{W}^{(j)}$ is arbitrarily chosen and the signals are nonorthogonal, we expect that in reality there will be multiple rows in any given subband for which $\mathbf{h}_{p}^{T} \mathbf{s}_{p}^{j}$ is nonzero, where $\mathbf{h}_{p}$ denotes the $p$ th column of $\mathbf{H}$. The goal is therefore to rank-order the subbands based on the degree to which they are able to preserve the signal subspace. This is feasible by rank-ordering the eigenvalues across subbands and examining their corresponding eigenvectors $\mathbf{U}_{X}^{j}$. Specifically, this can be achieved in two different ways.

(1) Within subband $j$, the blind source separation process amounts to finding the signal eigenvalues that correspond to the group of sources that possess nonzero projections onto the $j$ th wavelet basis, that is, $\mathbf{h}_{p}^{T} \mathbf{s}_{p}^{j}$ is nonzero for $p=1, \ldots, P_{j}$. These will be ranked in decreasing order of magnitude according to

$$
\begin{gathered}
\lambda_{1}^{j}>\lambda_{2}^{j}>\cdots>\lambda_{P_{j}}^{j} \Longleftrightarrow \mathbf{h}_{p_{1}}^{T} \mathbf{s}_{p_{1}}^{j}>\mathbf{h}_{p_{2}}^{T} \mathbf{s}_{p_{2}}^{j}>\cdots>\mathbf{h}_{P_{j}}^{T} \mathbf{s}_{P_{j}}^{j} \\
\text { such that } p_{1}=\underset{p \in\left\{1, \ldots, P_{j}\right\}}{\arg \max } \mathbf{h}_{p}^{T} \mathbf{s}_{p}^{j} .
\end{gathered}
$$

(2) Given a specific source $p^{*} \in\{1, \ldots, P\}$, the source classification process amounts to specifying an operator $B_{p^{*}}$, that finds the set of subband indices among all $j \in \Delta\{J\}$ for which there exist an invariant eigenvector $\mathbf{u}_{p^{*}}^{j}$. That is,

$$
\begin{aligned}
\lambda_{p}^{j_{1}}>\lambda_{p}^{j_{2}}>\ldots>\lambda_{p}^{J_{p}}>\Longleftrightarrow \mathbf{h}_{p}^{T} \mathbf{s}_{p}^{j_{1}}>\mathbf{h}_{p}^{T} \mathbf{s}_{p}^{j_{2}}>\ldots>\mathbf{h}_{p}^{T} \mathbf{s}_{p}^{J_{p}} \\
\text { such that } p^{*}=\underset{j \in \Delta\{J\}}{\arg \min }\left\|\mathbf{u}_{p^{*}}^{j}-\mathbf{a}_{p^{*}}\right\|^{2}
\end{aligned}
$$

\footnotetext{
${ }^{2}$ For a 2-band orthonormal discrete wavelet packet transform up to $L$ decomposition levels, a binary tree representation would consist of a total of $J=2^{L+1}-1$ subbands.
}

where $\mathbf{a}_{p *}$ denotes the $p^{*}$ th independent column of the matrix $A$. This set of basis, now labeled $\mathfrak{J}_{p^{*}} \subset \Delta\{J\}$, will constitute the "best basis" representing the source $p^{*}$.

\subsection{Best basis selection}

The second interpretation in (9) falls under the class of best basis selection schemes, originally introduced in [20]. The idea can be summarized as follows. In representing the discrete signal successively into different frequency bands in terms of a set of overcomplete orthonormal basis functions, one obtains a dictionary of basis to choose from. These are represented by a binary tree in which high amplitude wavelet coefficients in a certain node indicate the presence of the corresponding basis in the signal and measure its contribution. Equivalently, they evaluate the content of the signal inside the related frequency subband. Best signal representation is obtained by defining a cost function for pruning the binary tree. In [20], it was suggested to prune the tree by minimizing an entropy cost function between the parent and children nodes. The cost of each node in the binary tree is compared to the cost of its children. A parent node is marked as a terminal node if it yields a lower cost than its children cost. Other cost functions such as mean square error (MSE) minimization were suggested in [21]. Clearly, one cost function selection may be suitable for some signal types while not the best for others.

In our context, the cost function can be expressed in terms of the invariance property of the signal subspace $\{\mathbf{A}\}_{j}$ of children nodes compared to their parent node. Specifically, a child node is considered a candidate for further splitting if the Euclidean distance between the signal subspace in the parent node and that of the child is minimized. This can be expressed as

$$
\operatorname{cost}(j, p)=\min _{j \in \mathfrak{J}_{p}}\left\|\mathbf{u}_{p}^{\mathrm{j}=\text { Parent }}-\mathbf{u}_{p}^{\mathrm{j}=\text { Child }}\right\|^{2} .
$$

The cost definition ensures that for those children nodes that do not have a "similar" signal subspace to that of the parent, they will not be marked as candidates for further splitting. The search in the binary tree is performed in a topdown scheme, starting from the time domain signal matrix $\mathbf{Y}$ that is guaranteed to contain the full signal subspace $\{\mathbf{A}\}$. Generally speaking, wavelet coefficients exhibit large interscale dependency [22-24]. Therefore, it is anticipated that if the signal subspace is spanned by the wavelet basis in a parent node, it will be spanned by the wavelet basis of at least one of the children nodes.

\subsection{Noisy model}

Let us now consider the general observation model in the presence of additive noise. The observation matrix $\mathbf{Y} \in$ $\mathbb{R}^{M \times N}$ can be expressed as

$$
\mathbf{Y}=\mathbf{X}+\mathbf{Z}=\mathbf{A S}+\mathbf{Z}
$$

where $\mathbf{Z} \in \mathbb{R}^{M \times N}$ denotes a zero-mean additive noise with arbitrary spatial and temporal covariances $\mathbf{R}_{Z} \in \mathbb{R}^{M \times M}$ and 
$\mathrm{C}_{Z} \in \mathbb{R}^{N \times N}$, respectively. Using SVD, Y can be spectrally factored to yield

$$
\mathbf{Y}=\mathbf{U}_{Y} \mathbf{D}_{Y} Y_{V}^{T}=\sum_{m=1}^{M} \lambda_{m} \mathbf{u}_{m} \mathbf{v}_{m}^{T}
$$

where $\lambda_{m}$ denotes the $m$ th singular value corresponding to the $m$ th diagonal entry in $\mathbf{D}_{Y}=\operatorname{diag}\left[\lambda_{1} \cdots \lambda_{M}\right]$, and $\mathbf{U}_{Y}=$ $\left[\mathbf{u}_{1}, \mathbf{u}_{2}, \ldots, \mathbf{u}_{M}\right] \in \mathbb{R}^{M \times M}$ comprises the eigenvectors spanning the column space of $\mathbf{Y}$, while $\mathbf{V}_{Y}=\left[\mathbf{v}_{1}, \mathbf{v}_{2}, \ldots, \mathbf{v}_{N}\right] \mathbb{R}^{N \times N}$ comprises the eigenvectors spanning the row space of $\mathbf{Y}$. If $\mathbf{Y}$ is a linear mixture of $P$ orthogonal signal sources contaminated by additive white noise, then the first $P$ columns of $\mathbf{U}_{Y}$ will span the signal subspace $\{\mathbf{A}\}$, while the remaining $M-P$ columns of $\mathbf{U}_{Y}$ will span the orthogonal noise subspace $\{\mathbf{Z}\}$.

The matrix $\mathbf{Y}_{j}$ obtained through orthogonal transformation $\mathbf{W}^{(j)}$ can be likewise decomposed using SVD to yield

$$
\mathbf{Y}_{j}=\mathbf{A} \mathbf{S}_{j}+\mathbf{Z}_{j}=\mathbf{U}_{Y}^{j} \mathbf{D}_{Y}^{j} \mathbf{V}_{Y}^{j^{T}},
$$

where $\mathbf{Z}_{j}$ expresses the projection of the noise matrix onto the subspace $\Omega_{j}$. Similar to the analysis in the noise-free case, the span of $\mathbf{V}_{Y}^{j}$ directly impacts the span of the column space of $\mathbf{U}_{Y}^{j}$. However, this case is not trivial due to the presence of the noise since the eigenvalues $\lambda_{P_{j}+1}^{j}>\lambda_{P_{j}+2}^{j}>\cdots>\lambda_{M}^{j}$ are nonzero with probability one.

To make the presentation clear, let us consider the simplistic illustration in Figure 1. In this illustration, it is assumed that the dictionary obtained contains a total of three wavelet basis. For completeness, this implies that all the functions in $L_{2}(\mathbb{R})$ reside in the space spanned by the fixed bases $\boldsymbol{\beta}_{i}, \boldsymbol{\beta}_{l}$, and $\boldsymbol{\beta}_{k}$, respectively. The row space of $\mathbf{X}=\mathbf{A S}$, denoted $\{\mathbf{X}\}$, and the row space of $\mathbf{Z}$, denoted $\{\mathbf{Z}\}$, are projected onto this three-dimensional wavelet space. This representation permits visualizing how the projection of the noise row space $\{\mathbf{Z}\}$ results in two components, namely, $\{\mathbf{Z}\}_{/ /}$ that resides in the signal subspace (correlated noise component), and $\{\mathbf{Z}\}_{\perp}$ that is orthogonal to the signal subspace $\{\mathbf{X}\}$ (white noise component). In this representation, $\{\mathbf{Z}\}_{\perp}$ is spanned by the wavelet base $\boldsymbol{\beta}_{i}$. On the other hand, $\{\mathbf{Z}\}_{/ /}$ is spanned by $\boldsymbol{\beta}_{l}$ and $\boldsymbol{\beta}_{k}$, respectively. The projections of the noise $\{\mathbf{Z}\}_{/ /}$onto these bases are denoted $\{\mathbf{Z}\}_{l}$ and $\{\mathbf{Z}\}_{k}$, respectively. In a similar fashion, the signal subspace $\{\mathbf{X}\}$ can be projected onto the basis $\boldsymbol{\beta}_{l}$ and $\boldsymbol{\beta}_{k}$, resulting in the signal components $\{\mathbf{X}\}_{l}$ and $\{\mathbf{X}\}_{k}$, respectively. It is thus assumed that $\boldsymbol{\beta}_{i}$ does not represent any of the signal sources, that is, $\mathbf{H}^{\mathrm{T}} \mathbf{S}_{i}=0_{P \times N}$. Careful examination of these projections yields the following.

(1) Any signal projection that belongs to $\{\mathbf{X}\}_{l}$ is dominant over noise projections $\{\mathbf{Z}\}_{\text {l }}$.

(2) Any noise projection that belongs to $\{\mathbf{Z}\}_{k}$ is dominant over the signal projections $\{\mathbf{X}\}_{k}$.

(3) Any noise projection that belongs to $\{\mathbf{Z}\}_{\perp}$ is fully accounted for by the wavelet basis $\boldsymbol{\beta}_{i}$.

Therefore, the best basis set $\mathfrak{J}_{p}$ for source $p$ would contain only the index $l$. If $\{\mathbf{X}\}$ contained only a single source $p$, then the dominant eigenvalue $\lambda_{1}^{l}$ will correspond to the

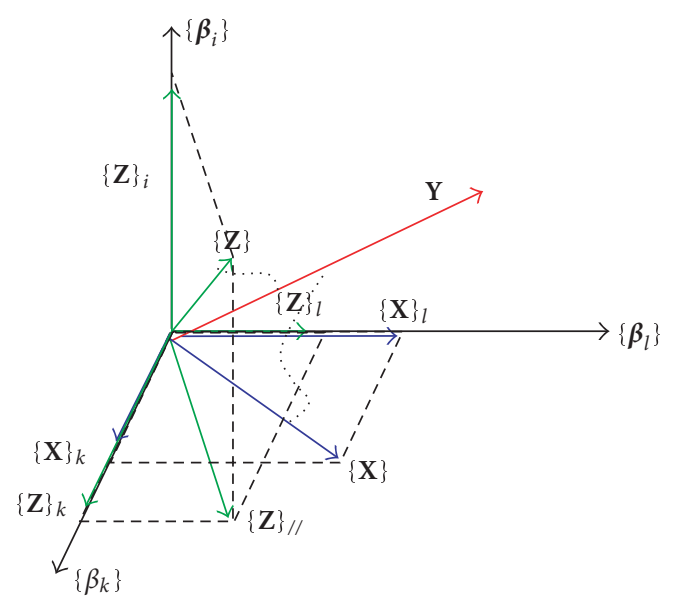

Figure 1: Projection of the signal and noise subspaces $\{\mathbf{X}\}$ (blue), and $\{\mathbf{Z}\}$ (green), respectively, onto a fixed orthogonal basis space. The space is assumed to be completely spanned by three orthogonal basis $\left\{\boldsymbol{\beta}_{l}\right\},\left\{\boldsymbol{\beta}_{\boldsymbol{k}}\right\}$, and $\left\{\boldsymbol{\beta}_{\boldsymbol{i}}\right\}$ for clarity.

eigenvector $\mathbf{u}_{1}^{l}$ spanning the signal subspace, which would be a $1 \mathrm{D}$ space spanned by the single column matrix $\mathbf{A}$.

The sparsity introduced by the orthogonal transformation again plays an important role in the noisy model. This is because the noise spreads out across resolution levels to many small coefficients that are easy to threshold using the denoising property of the DWT $[25,26]$. Therefore, the once ill-determined separation gap between the signal eigenvalues and those of the noise when the noise is caused by weak sources becomes relatively easier to determine. Thus the advantages gained by exploiting subspace decomposition in the transform domain become obvious. These are (1) reduction of the contribution of the unknown correlation coefficients $\rho_{i j}$ on the eigenvalues of the signal matrix $\mathbf{X}$, and (2) enhancing the separation gap between the signal and noise eigenvalues when the noise is correlated.

\subsection{Subband-dependent signal subspace dimension}

Generalizing the example in Figure 1 to an arbitrary number of wavelet basis in the dictionary obtained, we obtain a set of wavelet basis $\boldsymbol{\beta}_{l}$ for each source in which the signal subspace projection $\{\mathbf{X}\}_{l}$ dominates over the noise subspace projection $\{\mathbf{Z}\}_{l}$. These are denoted $\mathfrak{J}_{1}\{l\}, \mathfrak{J}_{2}\{l\}, \ldots, \mathfrak{J}_{P}\{l\} \subset \Delta\{J\} .^{3}$ We reiterate that since both the signal matrix and the mixing matrix are unknown, our interest is to separate the most dominant sources in the mixture. Due to nonzero correlation among signals, or when $P>M$, the problem becomes ill-posed. In that respect, the time domain model in (2) may over/underestimate the dimension of the signal subspace. However, with the transformed model in (6), the sparsity introduced by the DWPT considerably mitigates the effect of

\footnotetext{
${ }^{3}$ The index $l$ will be used thereafter to indicate the basis indices for which the signal subspace projection dominates over the noise subspace projection.
} 
signal correlation, which maximizes the likelihood of estimating the correct $P_{j}$. We have shown previously [19] that a multiresolution sphericity test can be used to determine $P_{j}$ by examining the ratio of the geometric mean of the eigenvalues, $\lambda_{m}^{j}$ 's, to the arithmetic mean as

$$
\Lambda_{j}=\frac{\left(\prod_{m=1}^{M} \lambda_{m}^{j}\right)^{(1 / M-i+1)}}{(1 /(M-i+1)) \sum_{m=i}^{M} \lambda_{m}^{j}}, \quad i=1, \ldots, M-1 .
$$

This test determines the equality of the smallest eigenvalues (presumably the noise eigenvalues), or equivalently how spherical the noise subspace is. It determines how many signal subspace components are projected onto the signal subspace. The test consists of a series of nested hypothesis tests [27], testing $M-i$ eigenvalues for equality. The hypotheses are of the form

$$
\begin{aligned}
H_{0}\left(P_{j}\right): \lambda_{1}^{j} & \geq \lambda_{2}^{j} \geq \cdots \lambda_{P_{j}+1}^{j} \\
& =\lambda_{P_{j}+2}^{j}=\cdots=\lambda_{M}^{j}, \\
H_{1}\left(P_{j}\right): \lambda_{1}^{j} & \geq \lambda_{2}^{j} \geq \cdots \lambda_{P_{j}}^{j} \\
& \geq \lambda_{P_{j}+1}^{j} \cdots>\lambda_{M}^{j},
\end{aligned}
$$

We are interested in finding the smallest value of $P_{j}$ for which the null hypothesis is true. Using a desired performance threshold for the probability of false alarm (over determination of $\left.P_{j}\right), P_{j}$ dominant modes are described by their corresponding rank ordered $P_{j}$ eigenvectors.

We should point out that there are multiple ways the algorithm can be implemented. We summarize below one possible implementation.

(1) Compute the orthogonal transformation of the observation matrix row wise up to $L$ decomposition levels.

(2) For each subband, compute the eigendecomposition of the sample covariance matrix of the transformed observation matrix.

(3) For each eigenmode, rank-order the subbands based on the magnitude of their eigenvalues relative to the 0th subband eigenvalue.

(4) For each of the rank-ordered subbands, calculate the distance between each eigenvector and the corresponding 0th subband eigenvector. If the distances computed fall below a prespecified threshold, mark this subband as a candidate node in the best basis tree $\mathfrak{J}_{p}$. Otherwise, discard the current node and proceed to the next rank-ordered subband.

(5) For each of the candidate nodes, proceed in a bottomup approach by examining the parent-child relationship between the node indices. ${ }^{4}$ Nodes that do not have a parent node as a member of the candidate nodes set are discarded from the set $\mathfrak{J}_{p}$.

\footnotetext{
${ }^{4}$ In a dual-band DWPT tree with linear indexing, a parent node with index $l$ has children indices $2 l+1$ and $2 l+2$, respectively.
}

The outcome of these steps will permit identifying the characteristic best basis tree for each of the $P$ sources. This implementation can be used to interpret the algorithm as a classifier since the signal's spatial, temporal, and spectral features are expressed in terms of estimates of the signal parameters $\lambda_{p}^{l}, \mathbf{u}_{p}^{l}$ for $l \in \mathfrak{J}_{p}$ and $p=1, \ldots, P$. If the sources are Gaussian distributed, then it can be shown that the estimated parameters are also multivariate normal distributed. Therefore they can be optimally classified using likelihood methods [28, 29]. This analysis is outside the scope of this paper and is reported elsewhere [11].

\subsection{Computational complexity}

For the sake of completeness, we discuss briefly the computational complexity of the algorithm. For an $M \times N$ matrix, a full DWPT computation can be done in $O(M N)$ using classical convolution based algorithms [30]. There are two ways by which one can reduce this figure. First, the signals observed are known to be 1st level lowpass, therefore restricting the initial DWPT tree structure to descendants of the first level lowpass expansion does not affect the performance, but reduces the DWPT computations by $50 \%$. Second, we have experienced with more efficient and faster lifting-based algorithms that allow inplace computations [31], for which computational complexity can be reduced by another $42 \%-50 \%$ depending on the filter length [32]. So the complexity would be $\sim O(M N)$ for the DWPT computation. On the other hand, SVD computation takes $O\left(M N^{2}\right)$ computations, which can be reduced to $O(M c N)$ computations, where $c$ denotes the average number of nonzero entries per column, considering that the data becomes relatively sparse after DWPT decomposition using the Lanczos method [33]. This figure can be further reduced if incremental SVD is used, which takes $O(M N)$ computations. Eigenvector distance calculations across $J$ subbands can be feasibly done with $J \times M$ computations. Thus the total computational complexity would be in the order of $O(M N+M(N+1))$, which shows that the algorithm is very efficient since computations scale linearly.

\section{RESULTS}

We implemented the proposed algorithm and tested its performance on neurophysiological recordings obtained with microelectrode arrays in the brain. In this specific application, an array of microelectrodes is typically implanted in the cortex to record neural activity from a small population of neural cells as illustrated in the schematic of Figure 2. The neural activity of interest consists of short duration signals (typically 1-2 ms in duration), often termed neural "spikes" (due to their sharp transient nature), that occur irregularly in the form of a spike train [9]. Each spike waveform is generated whenever the membrane potential exceeds a certain threshold. The probability of spike generation depends on the input the neuron receives from other neurons in the population [36]. Generally speaking, neurons belonging to the same population have near-identical waveforms 


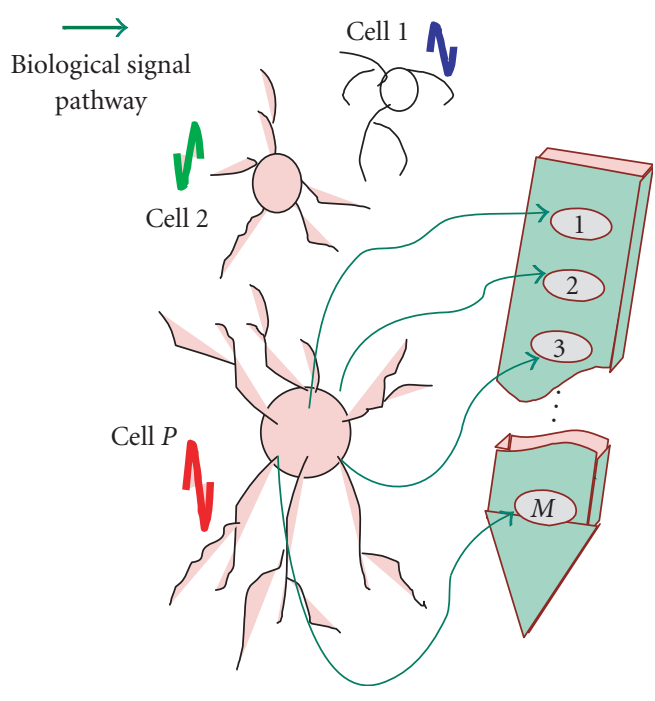

(a)

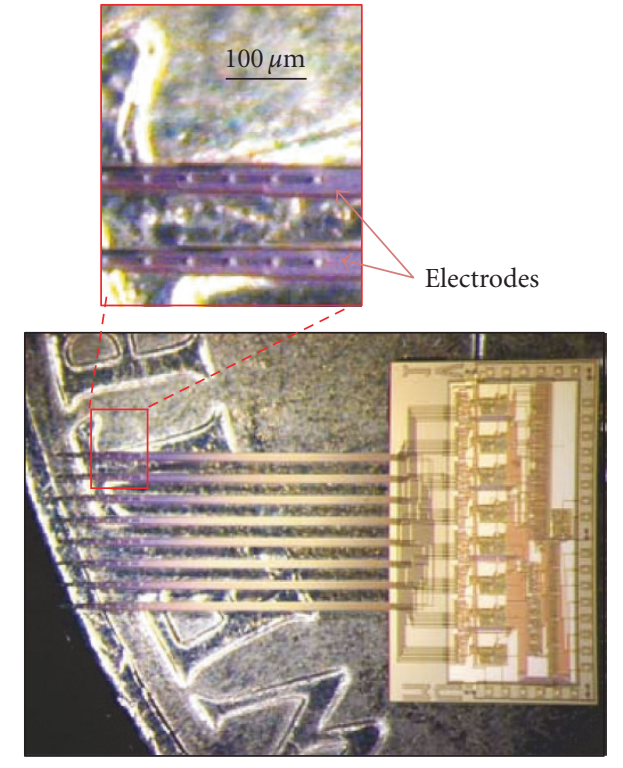

(b)

FIgURE 2: (a) Schematic of a microprobe array of $M$ electrodes monitoring neural activity from $P$ adjacent neural cells in the central nervous system. (b) A 64-channel Michigan electrode array with integrated electronics (amplification and bandpass filtering) on the back side of a US 1 cent [35].

at the source. However, due to many factors, the waveform from each neuron can be altered significantly due to the anisotropic properties of the transmission medium (extracellular space) [15]. The sensor array is generally designed to record the activity of a small population of neural cells in the vicinity of the array tip [35], thus the recordings are typically a mixture of multiple signal sources. The waveforms are generally distinct at the sensor array and can be used to discriminate between the original sources. However, significant correlation between the waveforms makes the separation task extremely complex [37], especially without prior knowledge of the exact waveform shape and the spatial distribution of the sources.

\subsection{Signal and noise characteristics}

To illustrate some characteristics of this signal environment with real data, typical neural signal characteristics are illustrated in Figure 3 for long data record as well as sample waveforms extracted from them in Figure 4. The spectral and spatial properties are also illustrated to demonstrate two important facts: first, the signals are wide-band, in the sense that the effective signal bandwidth is much larger than the reciprocal of the relative delay at which the signals are received at the different sensors or different times. Second, if the array is closely spaced, the signals tend to be largely coherent across multiple adjacent electrodes. Moreover, the noise spatial correlation extends over a much longer distance than the signal spatial correlation, which rolls off rapidly as a function of the distance between electrodes [10]. Sample spike waveforms are illustrated in Figure 4 to demonstrate their highly correlated nature among multiple sources. The shape of each waveform is a function of the source size, its distance from the array and the unknown variable conductivity of the extracellular medium $[15,38]$.

A firm understanding of the signal milieu reveals the following categorization of the noise sources.

(a) Thermal, electrical noise due to amplifiers in the headstage of the associated circuitry, and quantization noise introduced by the data acquisition system. This type can be regarded as a spatially and temporally white noise component belonging to the subspace $\{\mathbf{Z}\}_{\perp}$.

(b) High levels of background activity caused by sources far from the sensor array [39]. This noise type has spatially correlated components ranging from localized sources restricted to a subset of sensor array channels (can be regarded as weak interference sources) to far field sources engulfing the entire array. Both components belong to the subspace $\{\mathbf{Z}\}_{/ /}$.

\subsection{Features obtained}

We demonstrate two distinct signal sources along with their sample waveforms recorded on a 4-channel electrode array acquired experimentally in Figures 5 and 6, respectively. The observation matrix in each case contains a single source, thus $P=1$. We demonstrate in each figure the noisy spike waveform across channels along with its reconstructed waveform from the best basis [26]. In each case, the source feature set consists of the principal eigenmode $\left\{\lambda_{1}^{l}, \mathbf{u}_{1}^{l}\right\}$ across the best basis set $\mathfrak{J}_{1}$. 


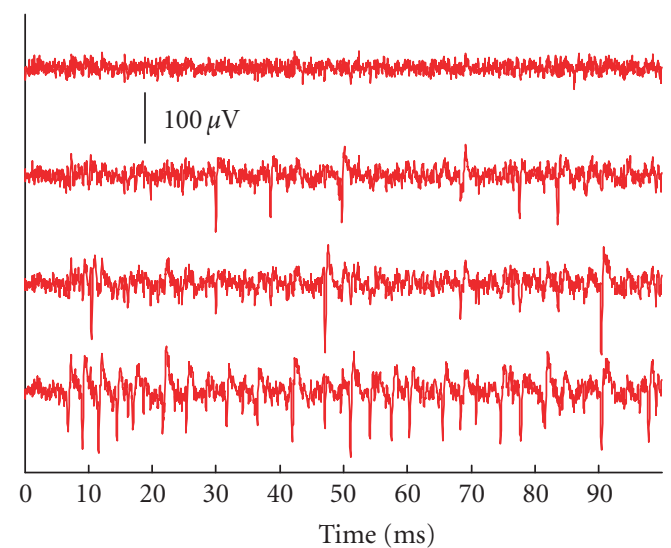

(a)

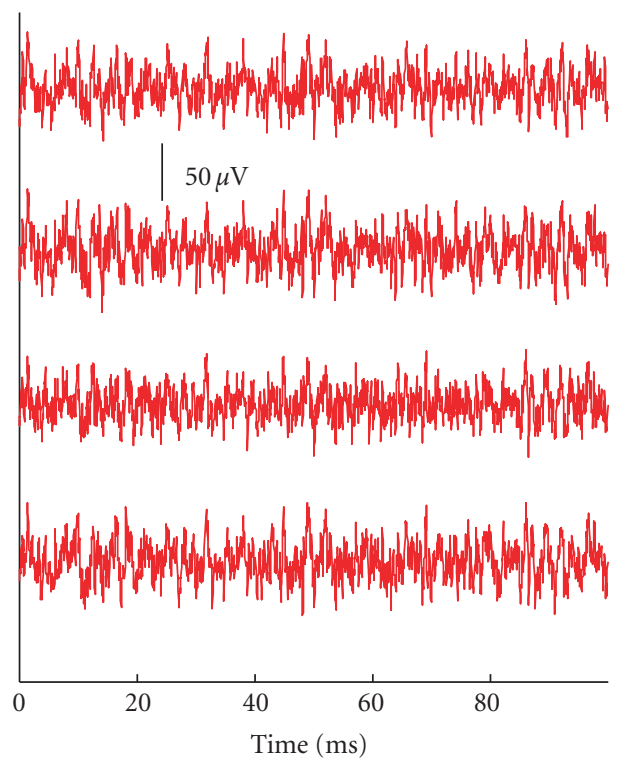

(c)

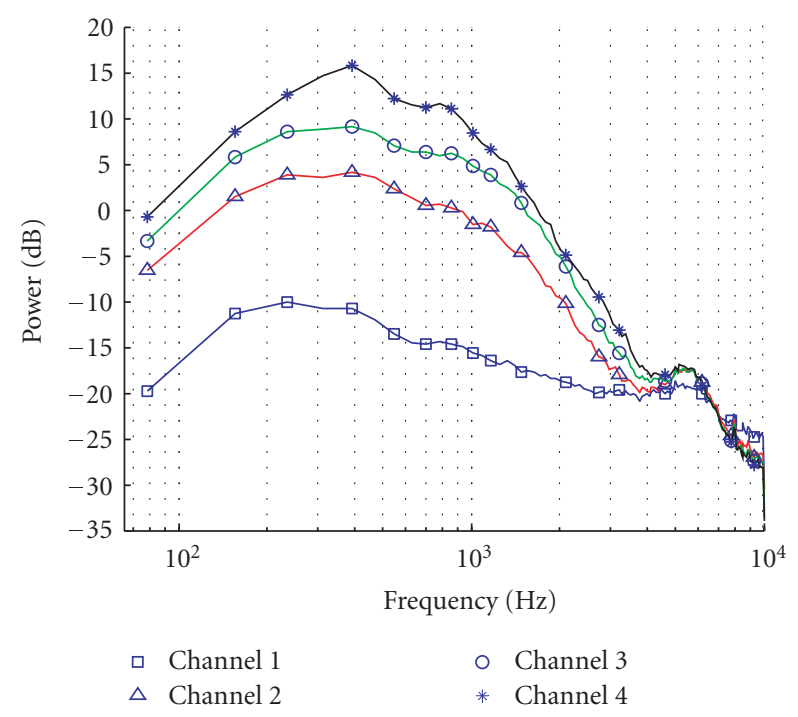

(b)

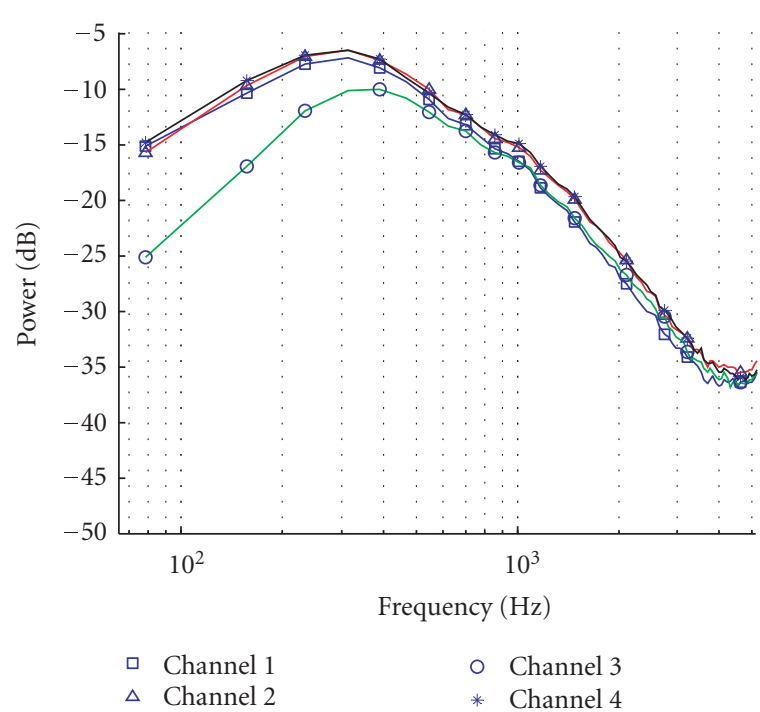

(d)

FIGURE 3: Characteristics of neural data measurements by a 4-electrode array. Data in (a) panel is considered high SNR signals (SNR $>4 \mathrm{~dB}$ ), while (c) panel is considered low SNR signals ( SNR $<4 \mathrm{~dB}$ ). The right panels illustrate the power spectral density of both data traces and show that most of the spectral content of the noise matches that of the signal within the $10 \mathrm{~Hz}-10 \mathrm{kHz}$ bandwidth but with reduced power indicating that neural noise constitutes most of the noise process.

As mentioned previously, zero-valued $\lambda_{1}^{l}$ indicates subband indices in which the $\ell_{2}$-norm of the signal subspace, in this case spanned by a single eigenvector $\mathbf{u}_{1}^{l}$, was not adequately preserved. This means that the cost in (9) was higher than the threshold needed to split the parent node. Note that we used a linear indexing scheme for labeling tree nodes for clarity. The averages displayed were calculated using a sample size of approximately 200 realizations of each source.
Figure 7 illustrates the case when two sources were present in the analysis interval. Careful examination of the compound waveform in Figure 7(c) reveals that some magnitude distortion occurs to source " $B$ " waveform (on channel 4 ) as a result of the overlap, while negligible distortion is noticed for source " $A$ " on channel 1 . This is because the signal subspace is clearly spanned by two distinct eigenvectors as indicated by the selection of columns of the mixing matrix as $\mathbf{a}_{1}=\left[\begin{array}{lllll}0.85 & 0.30 & 0.15 & 0.05\end{array}\right]$ and $\mathbf{a}_{2}=\left[\begin{array}{llll}0.05 & 0.10 & 0.20 & 0.80\end{array}\right]$. 

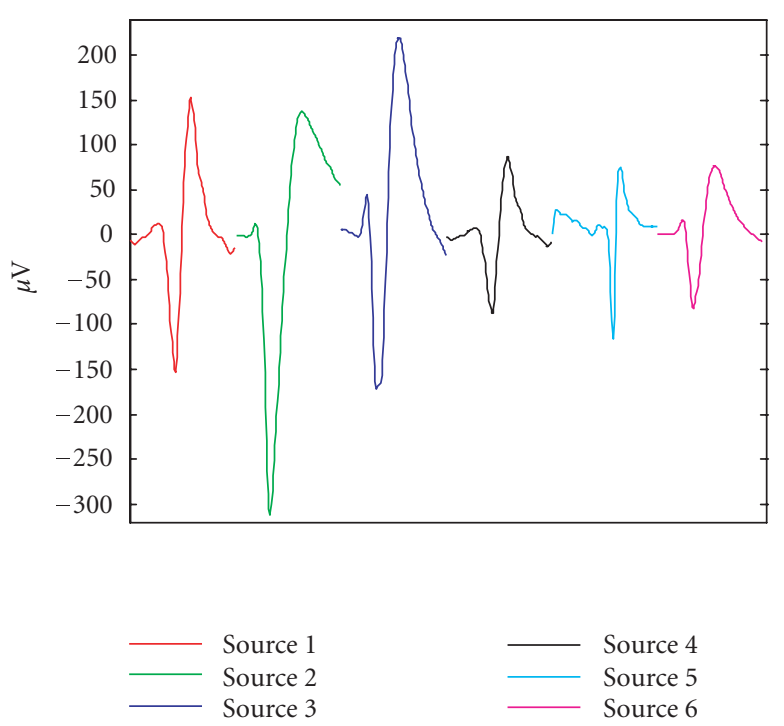

(a)

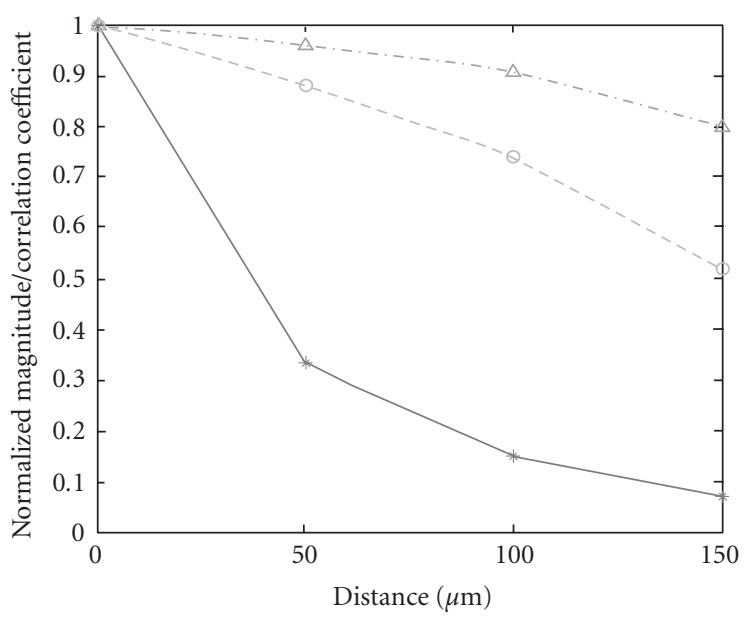

—* Spike amplitude

Noise correlation (stimulus)

$\triangle-$ Noise correlation (no stimulus)

(b)

FIGURE 4: Temporal and spatial characteristics of the observed signal and noise processes. The left panel demonstrates six waveforms extracted from recordings of six distinct neurons. Waveforms have been cleaned by proper time alignment and averaging across multiple realizations to display the templates shown.

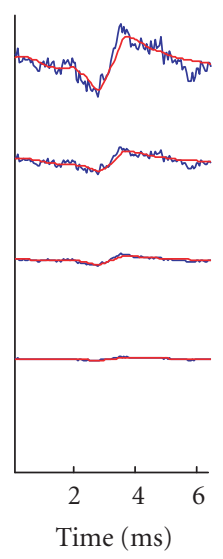

- Observed

— Reconstructed

(a)

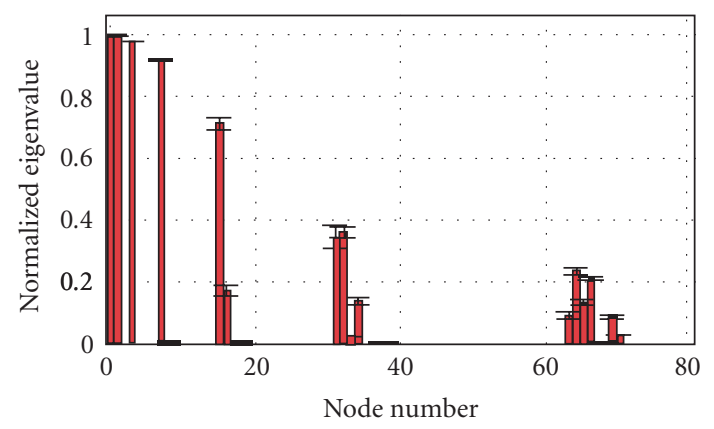

(c)

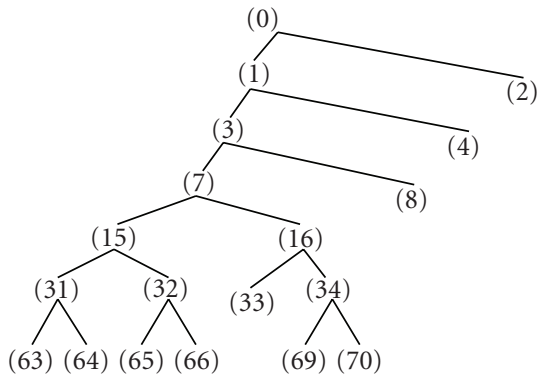

(b)

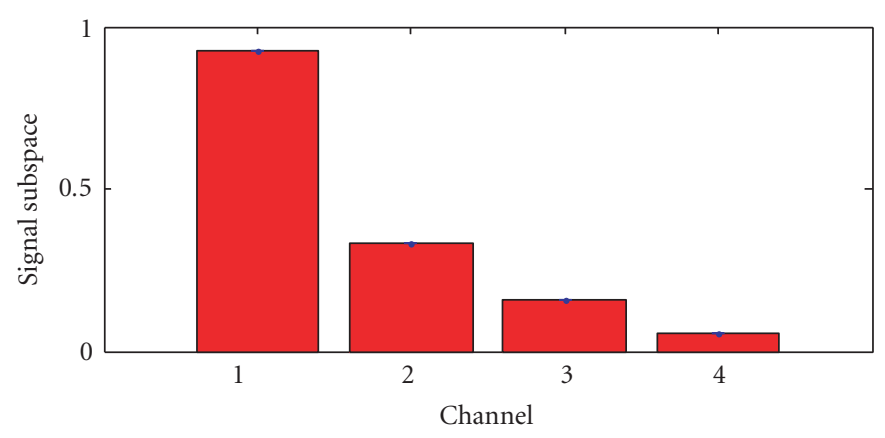

(d)

FIGURE 5: (a) Single realization of a signal from source 1 along a 4 -electrode array before and after best basis reconstruction $(\mathrm{SNR}=4 \mathrm{~dB}$ and $10.8 \mathrm{~dB}$, resp.). (b) Characteristic best basis wavelet packet tree (wavelet basis used was symlet of order 4). (c) Feature vector comprising sample mean of $\lambda_{1}^{l}$ for 200 realizations (standard deviation is shown as error bars). (d) Sample mean of the principal eigenvector $\mathbf{u}_{1}^{l}$ across best tree nodes for the realization in (a). 


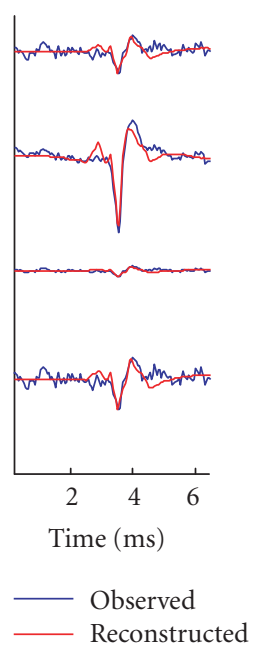

(a)

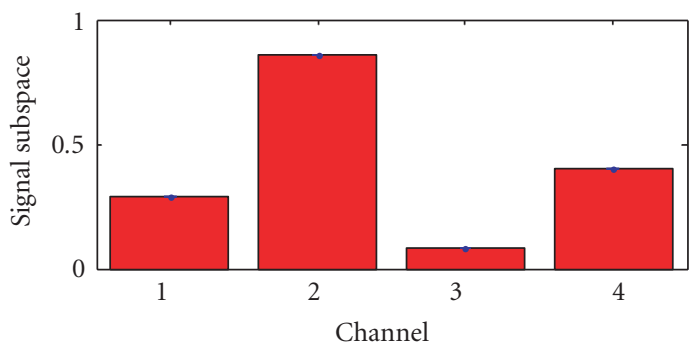

(c)

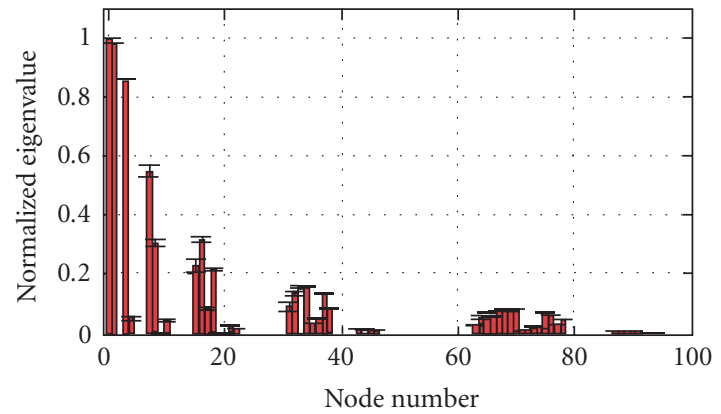

(b)

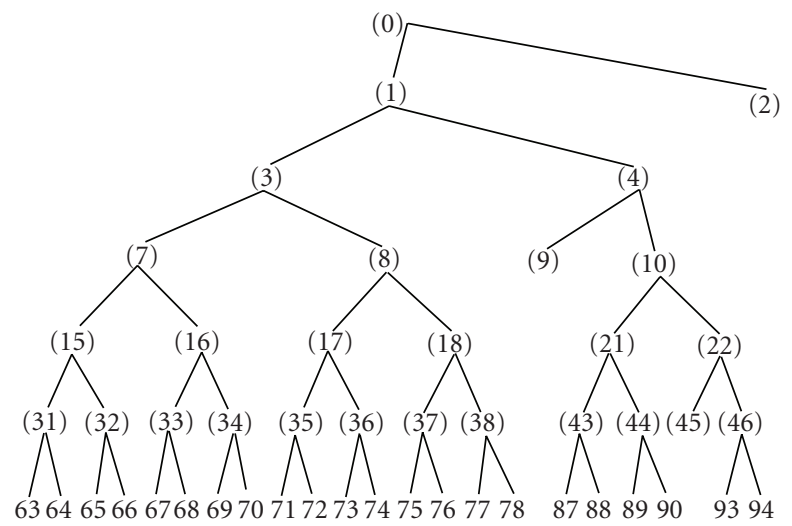

(d)

FIGURE 6: (a) Single source waveform along a 4-electrode array before and after best basis reconstruction ( $\mathrm{SNR}=5.7 \mathrm{~dB}$ and $10.8 \mathrm{~dB}$, resp.). (b) Feature vector comprising sample mean of $\lambda_{1}^{l}$ for 200 realizations, standard deviation is shown as error bars. (c) Sample mean of the principal eigenvector $\mathbf{u}_{1}^{l}$. (d) Characteristic best basis wavelet packet tree.

The first eigenmode $\left\{\lambda_{1}^{l}, \mathbf{u}_{1}^{l}\right\}$ is illustrated in Figure 7 in two different ways. First, in Figure 7(d) the mode is displayed across subbands similar to Figures 5 and 6. In Figure 7(e), the eigenmode is displayed by reindexing the nodes based on the decreasing order of magnitude of the eigenvalue $\lambda_{1}^{l}$. The purpose is to demonstrate how a threshold for $\lambda_{1}^{l}$ can be selected such that the set $\mathfrak{J}_{1}$ can be determined. As indicated by the MSE plot in Figures 7(d) and 7(e), the last node, say $j^{*}$, for which the cost (9) is below a predetermined threshold determines the minimum eigenvalue (dotted line in Figure 7(e), top panel) that corresponds to a signal component. It is clear that some nodes with indices $j<j^{*}$ in the ordered set (Figure 7(e), middle) do not correspond to a minimum MSE. These nodes have eigenvalues $\lambda_{1}^{j}>\lambda_{1}^{l^{*}}$ but their bases do not span the signal subspace. This is expected since these bases span the subspace of the correlated component of the two signals, which is stronger in these nodes such that the dominant eigenvector points in the direction of this component. These are eventually discarded from the set $\mathfrak{J}_{1}$.

Due to the sparsity introduced by the DWPT, the remaining nodes in Figure 7(e) can be clearly seen to span the subspace of source " $B$." These nodes have eigenvalues that are very close to zero as determined by the rank ordered $\lambda_{1}^{j}$ in the top panel and correspond to maximum MSE. This observation can be further made by examining Figure 8 in which the second eigenmode for the data matrix in Figure 7(c) is illustrated.

The interpretation of these observations is fairly straightforward: the set $\mathfrak{J}_{1}$ is dominated by the 1 st eigenmode, while the remaining nodes with indices $j \notin \mathfrak{J}_{1}$ consist of two subsets: one subset for which $\lambda_{1}^{j}$ is nonzero corresponds to basis spanning the "common" subspace of the two correlated signals. The other subset corresponds to the other source, 


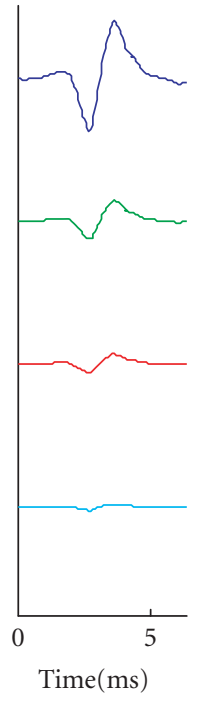

(a)

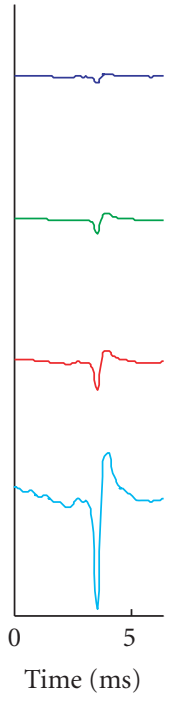

(b)

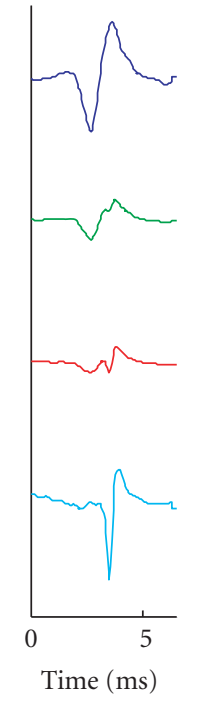

(c)
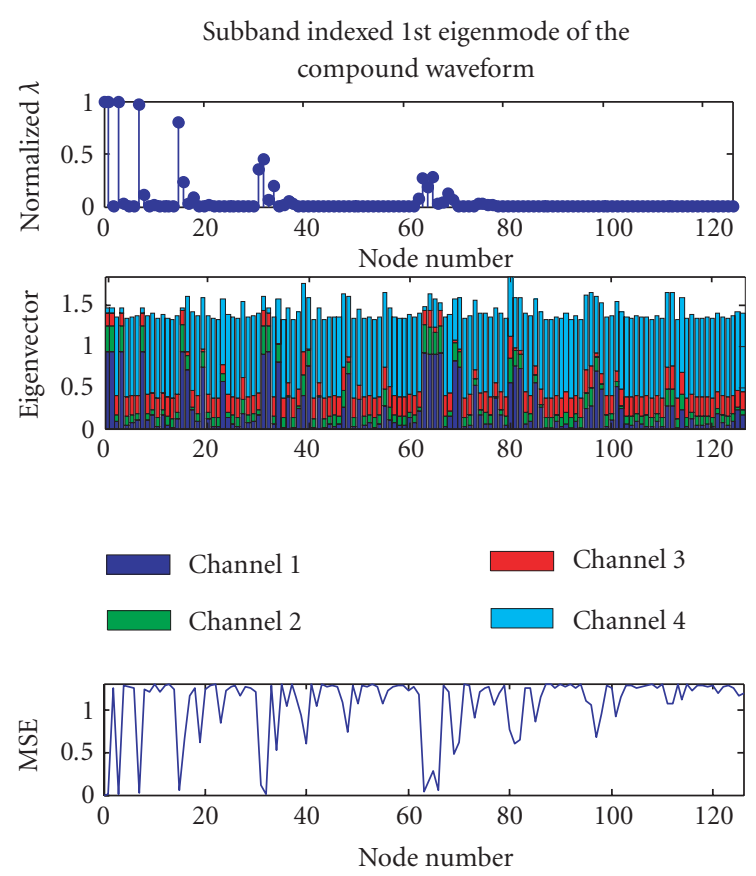

(d)
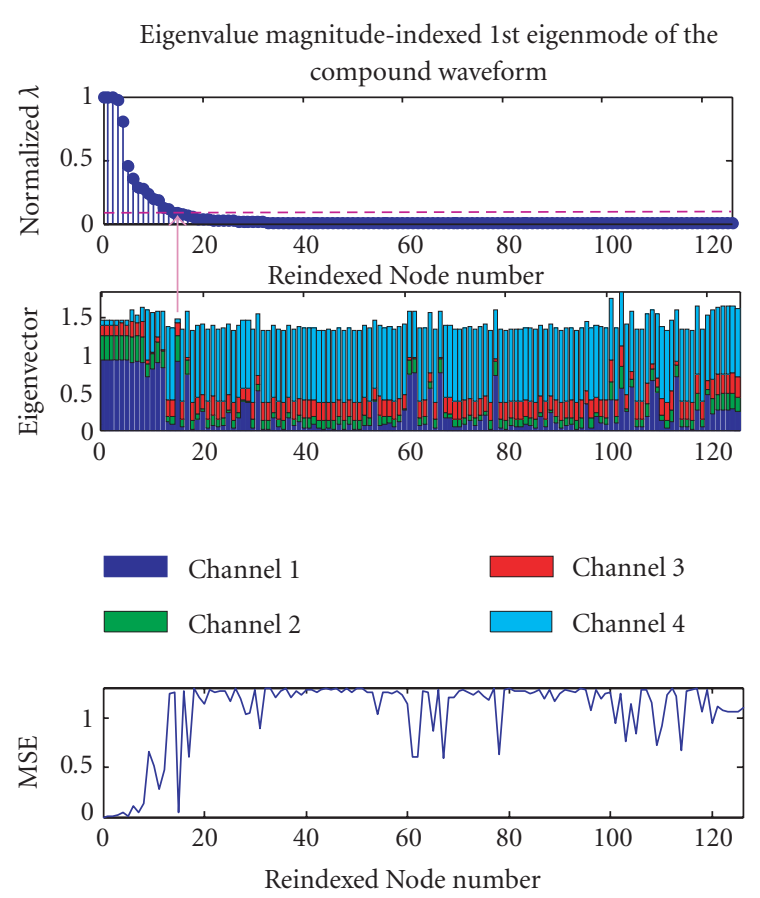

(e)

FIgURE 7: (a), (b) Template sources " $A$ " and " $B$ " across four channels with distinct mixing. Noise was filtered by averaging detected waveforms across 200 realizations. (c) Compound waveform obtained by summing the two signal matrices in (a) and (b). (d) 1st eigenvalue (top) and eigenvector (middle) mode of the compound waveform across the DWPT tree. Most of the nonzero eigenvalues correspond to eigenvectors that are similar to $\mathbf{u}_{1}^{0}$. In the bottom is the MSE between $\mathbf{u}_{1}^{0}$ and $\mathbf{u}_{1}^{j}, j \neq 0$. It is clear that the error reaches a minimum in nodes dominated by source " $A$." (e) Same data in (d) based on arranging $\lambda_{1}^{j}$ in descending order of magnitude. This allows identifying a threshold for $\lambda_{1}^{l}$ (as indicated by the arrow) that coincides with the last node $j^{*}$ having a minimum distance $\left\|\mathbf{u}_{1}^{j^{*}}-\mathbf{u}_{1}^{0}\right\|$.

which is regarded by the algorithm as "noise." This interpretation comes in perfect agreement with the vector space interpretation described in Figure 1.

We have further examined the more complex case where the signal subspaces are close. We selected $\mathbf{a}_{1}=$ $\left[\begin{array}{llll}0.82 & 0.15 & 0.31 & 0.05\end{array}\right]$ and $\mathbf{a}_{2}=\left[\begin{array}{llll}0.82 & 0.15 & 0.11 & 0.05\end{array}\right]$. Figure 9 illustrates the features obtained. In this case, we expected that the principal eigenmode $\left\{\lambda_{1}^{l} / \mathbf{u}_{1}^{l}\right\}$ will alternate between the two sets $\mathfrak{J}_{A}$ and $\mathfrak{J}_{B}$ (we used $p=1$ to indicate source " $A$ " and $p=2$ to indicate source 

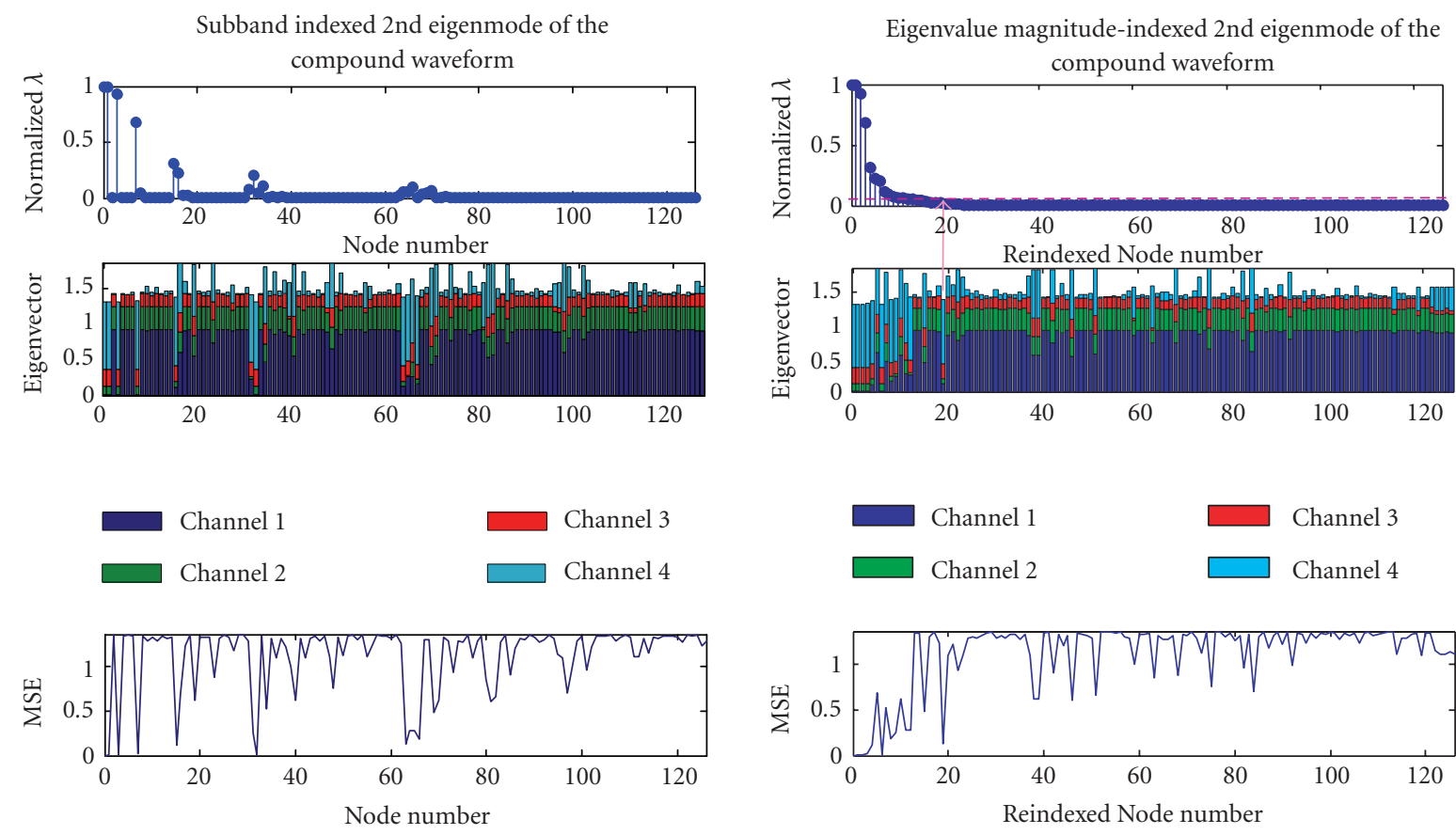

(a)
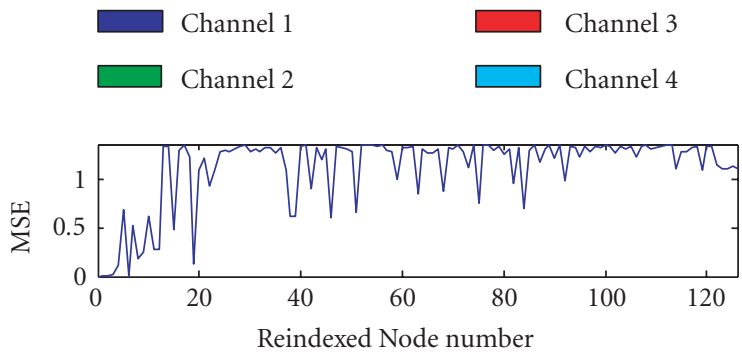

(b)

Figure 8: (a) Second eigenmode of the compound waveform in Figure 7(c). The mode is arranged similar to Figure 7(d) based on linear subband indexing. (b) Same features in (a) but reindexing the nodes based on descending order of magnitude of $\lambda_{2}^{j}$. This allows identifying a threshold for $\lambda_{2}^{l}\left(l \in \mathfrak{J}_{2}\right.$, as indicated by the arrow) that coincides with the last node $j^{*}$ in the sorted sequence having a minimum distance $\left\|\mathbf{u}^{j^{*}}-\mathbf{u}_{2}^{0}\right\|$.

" $B$ " for clarity of notation) depending on which basis best approximates the source with highest subband variance. Specifically, let us consider the set of ordered nodes for which $\lambda_{1}^{j}$ is nonzero. This includes roughly the ordered nodes 0 to 22. In Figure 9(e) (middle panel), this set of nodes can be divided in two subsets. The first subset (includes ordered nodes $\{0,1,2,3,4,5,6,9,10,13,15,18,22\}$ ) corresponds to an eigenvector spanning the same subspace as $\mathbf{a}_{1}$. The second subset (which includes ordered nodes $\{7,8,11,12,14,16,17,19,20,21\})$ corresponds to the eigenvector spanning the same subspace as $\mathbf{a}_{2}$. Mapping these nodes back to their original linear indexing in the binary tree yields the sets $\mathfrak{J}_{A}=\{0,1,3,7,15,16\}$ and $\mathfrak{J}_{B}=\{4,8,10,17,18,21\}$. Examining the tree structure in Figure 9(f) illustrates that the nodes in each set follow a parent-children relationship. Moreover, comparing these nodes to the individual best basis trees in Figures 5(b) and $6(d)$ for each individual source reveals that node indices belonging to $\mathfrak{J}_{B}$ are the ones that belong to source " $B$ " and do not belong to those of source " $A$."

The second eigenmode illustrated in Figure 10 corresponds to the direction where the difference between the signals is maximized. Specifically, let us examine each entry in the eigenvectors displayed in the middle panel of Figure 10(b). Since unequal mixing was introduced only on channel $3\left(\mathbf{a}_{1}[3] \neq \mathbf{a}_{2}[3]\right), \mathbf{u}_{2}[3]$ is the largest entry. This is expected since the second eigenvector should always point in the direction of maximum difference between the signals.
In addition, $\mathbf{u}_{2}[1]$ is nonzero because there is a difference in the $\mathfrak{l}_{2}$-norm of the original signals on channel 1 (Figures 9(a) and $9(\mathrm{~b})$ ). Entry $\mathbf{u}_{2}$ [2] (green) can be distinguished clearly in nodes forming elements of the set $\mathfrak{J}_{A}$, that is, those that are dominated by source " $A$," while this is not the case for the set $\mathfrak{J}_{B}$. This can be interpreted as channel 2 being the 3rd most discriminating feature between the shapes of sources " $A$ " and " $B$," after the unequal mixing on channel 3 and the distinct norms on channel 1, respectively. Lastly, $\mathbf{u}_{2}[4]$ is almost zero since both signals are very weak on channel 4 and are equally mixed.

\subsection{Consistency and robustness}

To assess the consistency and the robustness of the estimated parameters, a simulation was carried out using the spike templates illustrated in Figure 11 in which five distinct sources extracted from an array of four electrode channels using proper spike alignment and averaging are displayed. From this data, the "experimental" mixing matrix A could be readily obtained. We used these templates to simulate a data set with experimental noise extracted from the same recordings. The spike train of each source was obtained from a nonhomogenous Poisson process specifying the spike arrival time stamps for each source [9]. These were stacked in the rows of the signal matrix $\mathbf{S}$, then premultiplied by A. Noise variance was estimated from spike-free data from the same experiment. Then, data below the noise variance 


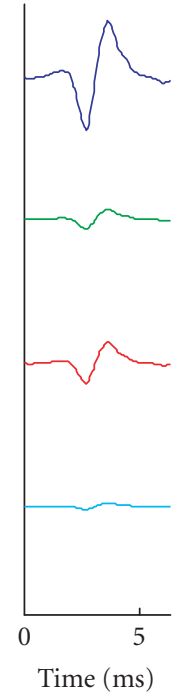

(a)

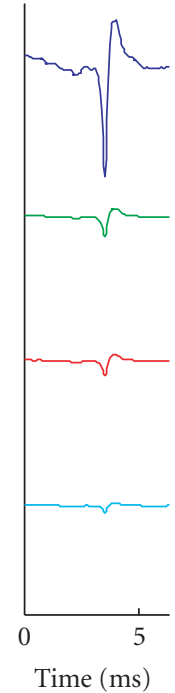

(b)

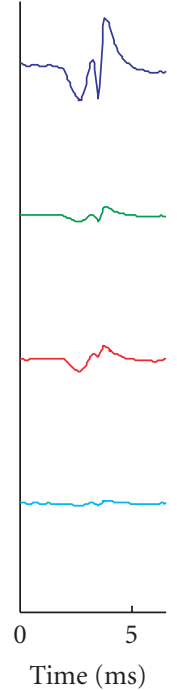

(c)
Subband indexed 1st eigenmode of the compound waveform
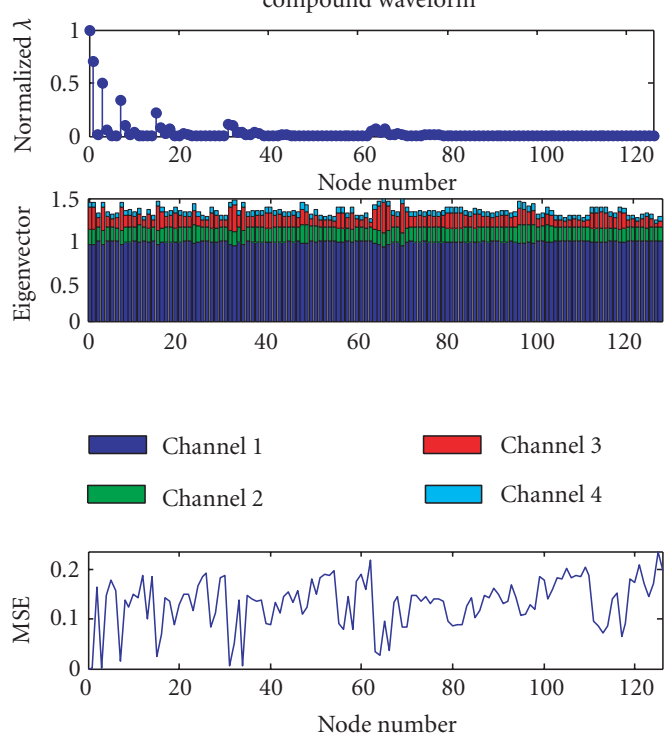

(d)
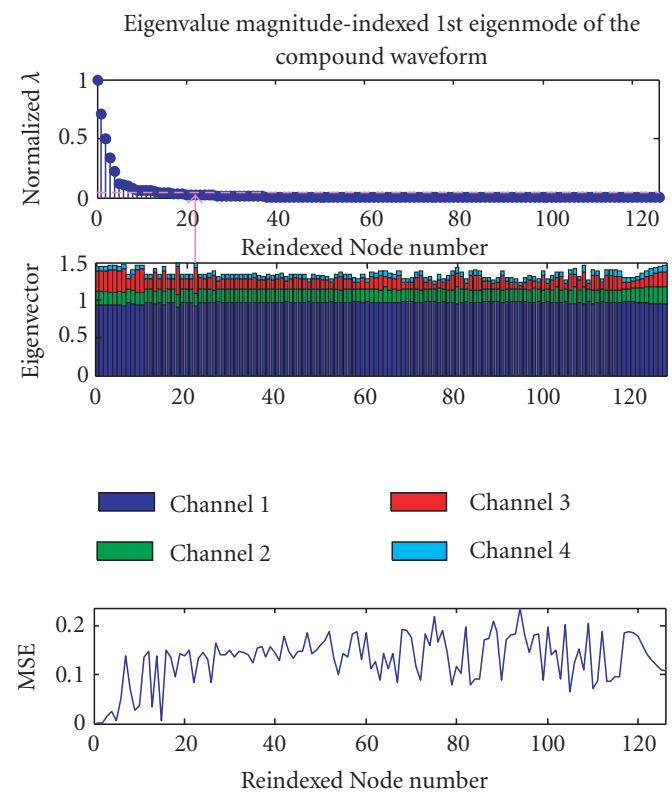

(e)

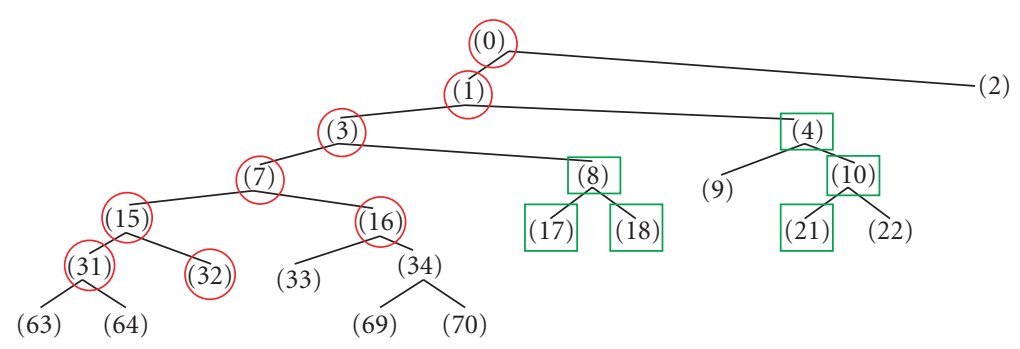

(f)

Figure 9: (a), (b) Template sources " $A$ " and " $B$ " with similar mixing, except for entry $\mathbf{a}_{1}[3] \neq \mathbf{a}_{2}$ [3]. (c) Compound waveform obtained by summing the two normalized signal matrices in (a) and (b). (d) 1st eigenmode of the compound waveform across the DWPT tree. The nonzero eigenvalues correspond to eigenvectors that alternate between sources $A$ and $B$ subspaces. (e) 1st eigenmode of the compound waveform displayed based on sorting $\lambda_{1}^{j}$ in descending order of magnitude. The set of ordered nodes up to node 22 can be seen to contain two subsets, each subset has eigenvectors spanning the subspace of each of the two sources. The MSE in the bottom panel was calculated with respect to $\mathbf{a}_{1}$. (f) Best basis binary tree using the first few eigenvalue ordered nodes. Nodes belonging to $\mathfrak{J}_{A}$ are circled, while those belonging to $\mathfrak{J}_{B}$ are boxed. 

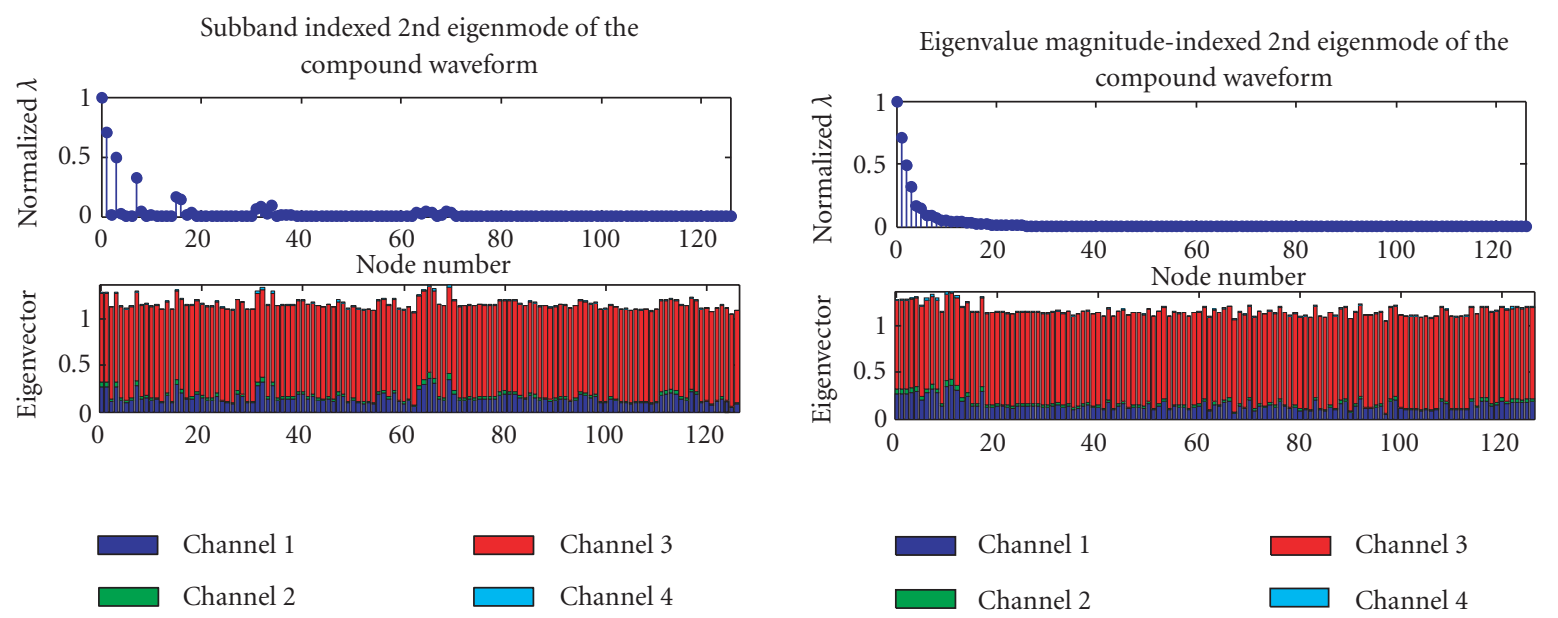

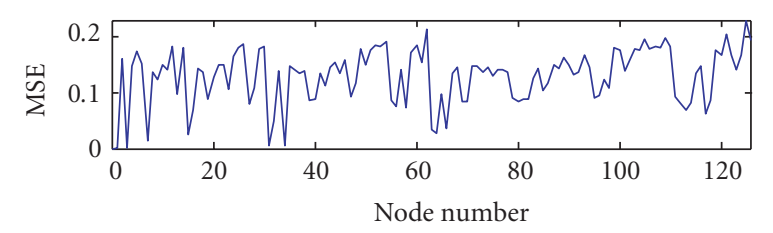

(a)

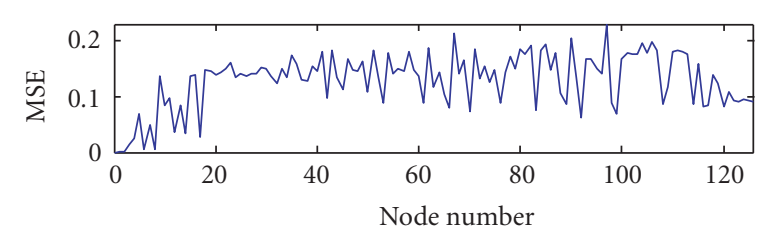

(b)

FIGURE 10: (a) 2nd eigenmode of the compound waveform in Figure 9(c). (b) Same data in (a) arranged in descending order of magnitude of $\lambda_{2}^{j}$.

was considered the colored noise component in the model described in (1) and was superimposed on the product AS yielding the observation matrix $\mathbf{Y}$.

The conventional definition of SNR in neurophysiological recordings is

$$
\operatorname{SNR}(\mathrm{dB})=20 \log _{10} \frac{\text { Signal peak to peak amplitude }}{\text { Noise RMS }} \text {. }
$$

As mentioned previously, the nature of the neural spikes is transient like, that is, comprises short duration events with sharp edges of different magnitude and waveform shape depending on the neural source relative location to the sensor array. The numerator is calculated by estimating the average peak-to-peak amplitude of spike waveforms detected on each channel. The denominator is calculated from spike-free data intervals as the noise root mean square. This helped assessing the robustness of the algorithm for variable SNR.

We varied the sample size and the SNR. In Figure 12, estimates of the signal subspaces are illustrated in (a). The variance of the estimates versus SNR for fixed sample size is illustrated in (b) for each source across channels. Note that the variance was too small so it is plotted in $\mathrm{dB}$ for clarity. It is clear that even in low SNR, the estimates exhibit very small variance $(\sim-50 \mathrm{~dB})$ implying the robustness of the estimates to degradation in SNR. The variances of the estimated eigenvalues versus sample size are illustrated in Figures 12(c) and 12(d) for each source across channels. It is obviously clear how a relatively small sample size of each source leads to a robust estimation of the eigenvectors given the observed small variance. The MSE between the true signal subspace and the estimated one is illustrated in Figure 12(b) for different SNRs and various sample sizes for each source. At low SNR (up to $4 \mathrm{~dB}$ ), the small sample size seems to affect the estimates, but consistency is clear when the sample size increases beyond 50 samples/source. On the other hand, at high SNR (above $6 \mathrm{~dB}$ ), the sample size does not seem to affect the MSE at all and seems to attain the irreducible error fairly quickly.

In Figure 13, we illustrate the variance of the eigenvalue estimates across best basis nodes versus sample size. It can be noticed that some nodes are sensitive to the small sample size; while others do not seem to be affected and achieve convergence relatively fast. The surprising result was the stability of some nodes at coarse scales (tree bottom) even though their estimated eigenvalues are relatively the smallest entries in the eigenvalue set $\lambda_{1}^{l}, l \in \mathfrak{J}_{p}$ (refer to Figure 6(b) for an example). This is very clear for source $B$ for instance, which was used in the example illustrated in Figure 6(a). On the other hand, the SNR seems to affect the eigenvalue estimates to some extent. This is clear from Figure 11(b), where the variance of the eigenvalue estimates is illustrated versus best tree nodes for different SNR.

To summarize, the results show consistency of the estimates as the sample size becomes large. The robustness of the estimates is exceedingly high especially those of the signal subspace and does not seem to be affected by the sample size. Accordingly, one may argue that the separation process can rely to a high degree of confidence on the signal subspace 

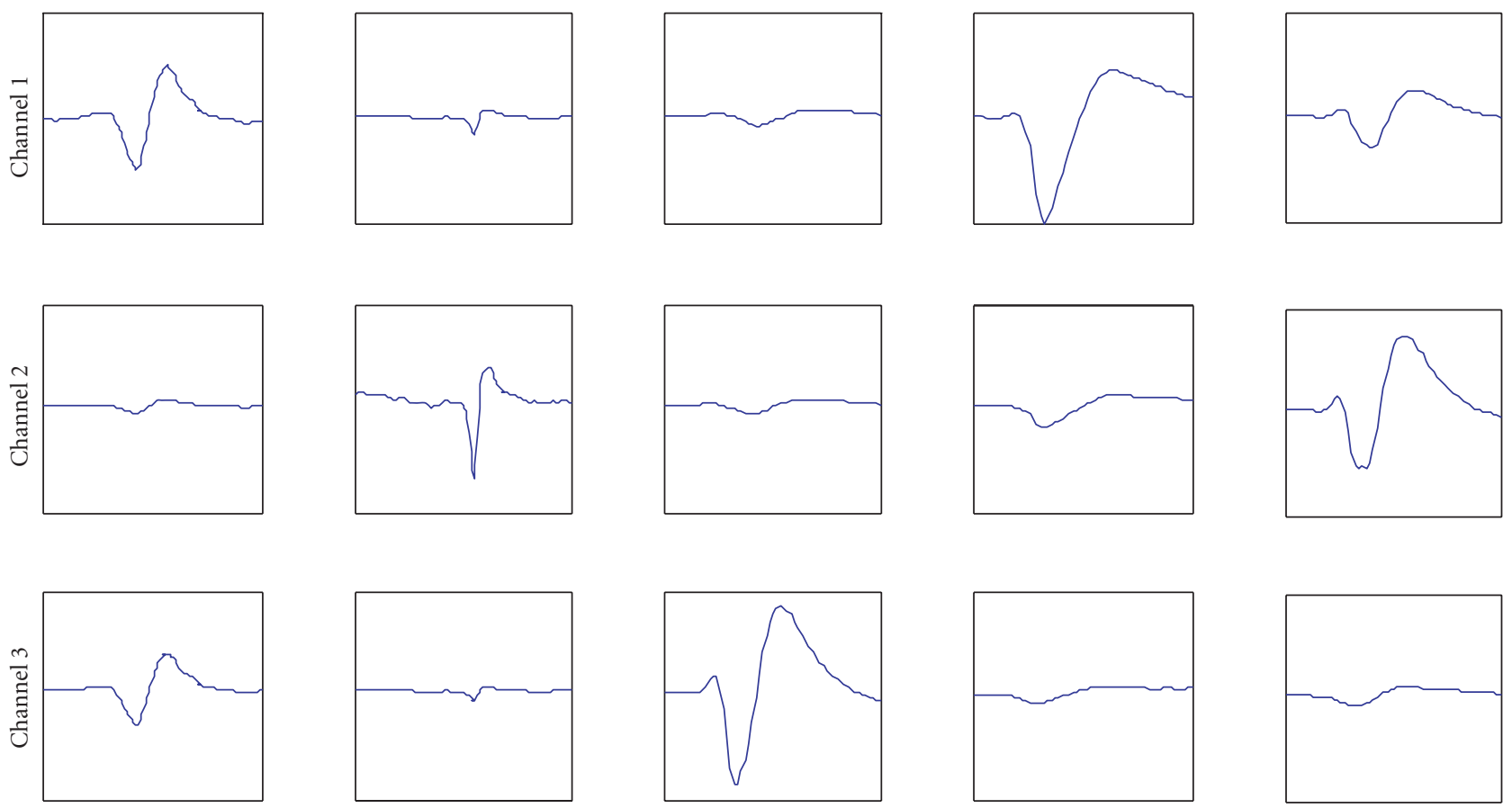

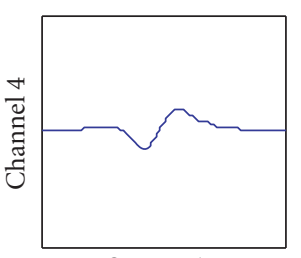

Source $A$

(a)

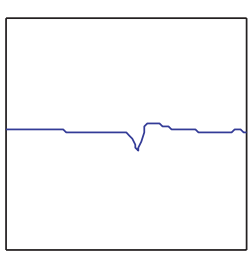

Source $B$

(b)

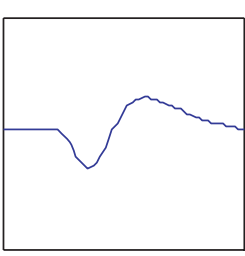

Source $C$

(c)

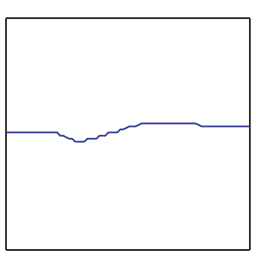

Source $D$

(d)

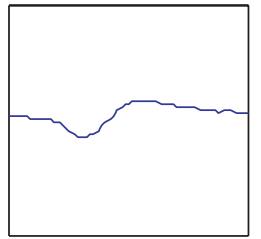

Source $E$

(e)

FIGURE 11: Template waveforms for 5 neural sources extracted from experimental data used to assess the consistency of the estimated parameters.

estimates in low SNR environments, since less variance of the estimates is noticed. For example, from Figures 12(c) and $12(d)$, it is apparent that the variance converges rapidly to the quantization noise level when the sample size is roughly around $20 \sim 25$ samples/source for an SNR $=2 \mathrm{~dB}$. Moreover, the observed variance can give some insight on the level of classification error for a given spike sample size. On the other hand, eigenvalues tend to be more stable when the SNR is moderate to high regardless of the sample size.

\subsection{Source detection}

We have investigated the performance of the sphericity test in (14) and compared it to classical source detection techniques such as the AIC and MDL [40] in the context of array processing $[41,42]$. Figure 14 illustrates the performance at multiple SNRs. We point out that one advantage gained using the multiresolution sphericity test is the tolerance it allows for erroneous decisions. Specifically, if a noise component predominates in a certain subband to the extent of masking a weak source, then the sphericity test may underestimate $P$ in that subband. In this case, the overall estimate of $P$ is minimally affected due to the fact that this source may be better represented in another subband where the noise has less masking effect thereby yielding a stronger mode that will depend on how much correlation exists between that source and the corresponding wavelet basis. The thresholds for the sphericity tests are determined using the fact that the likelihood function in (14) statistically approaches a Chi-square distribution with $(M-p)^{2}-1$ degrees of freedom [19].

\subsection{Invariance to temporal nonstationarity}

A well-known characteristic of neurophysiological recording is the nonstationarity in the signals over short time periods. This potentially occurs during bursting activity where it was shown that the signal waveform can exhibit more than 50\% reduction in amplitude [43]. Typically, neural sources firing successive spikes in a short period of time tend to introduce observable attenuation in spike waveform shape due to the 

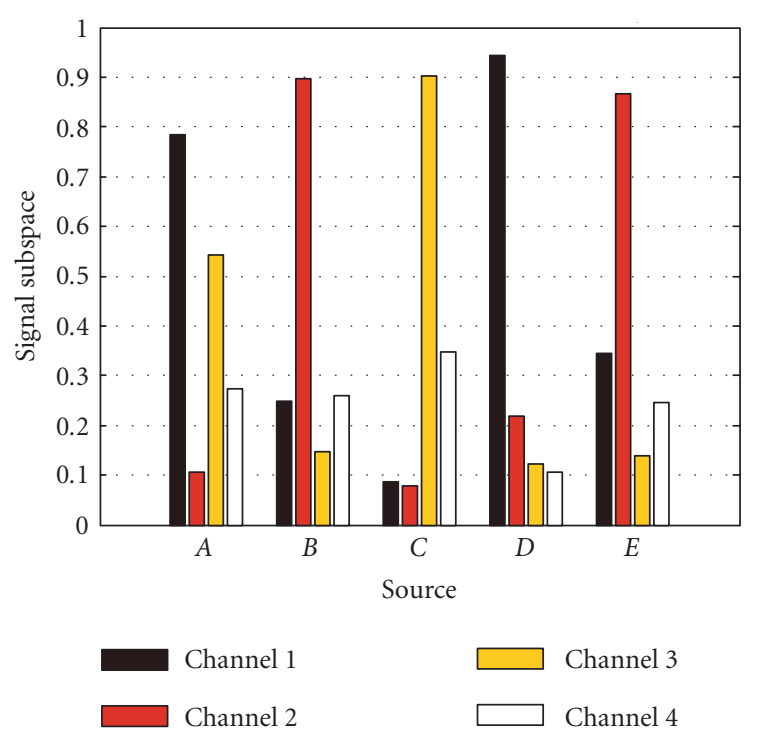

(a)

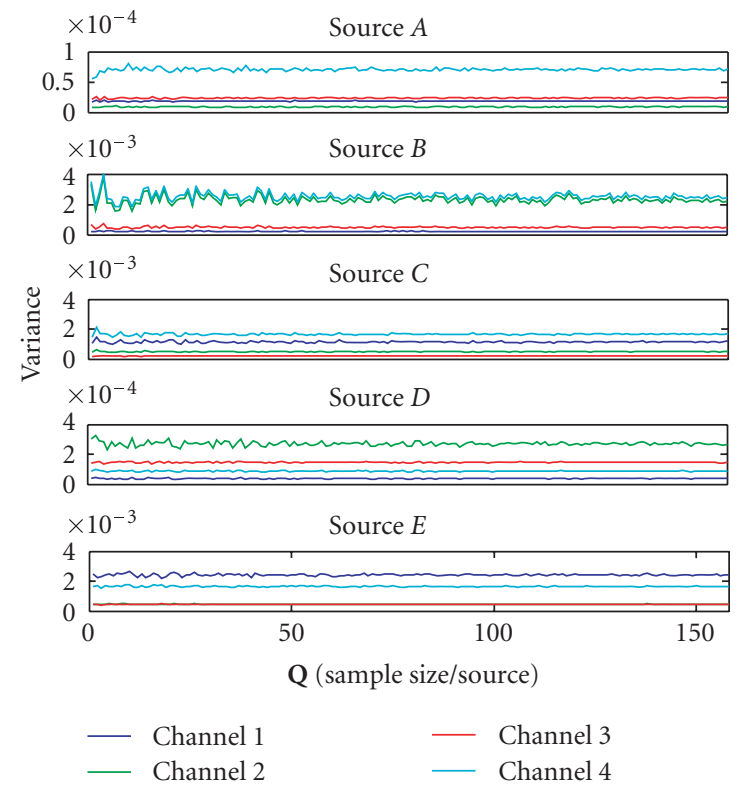

(c)
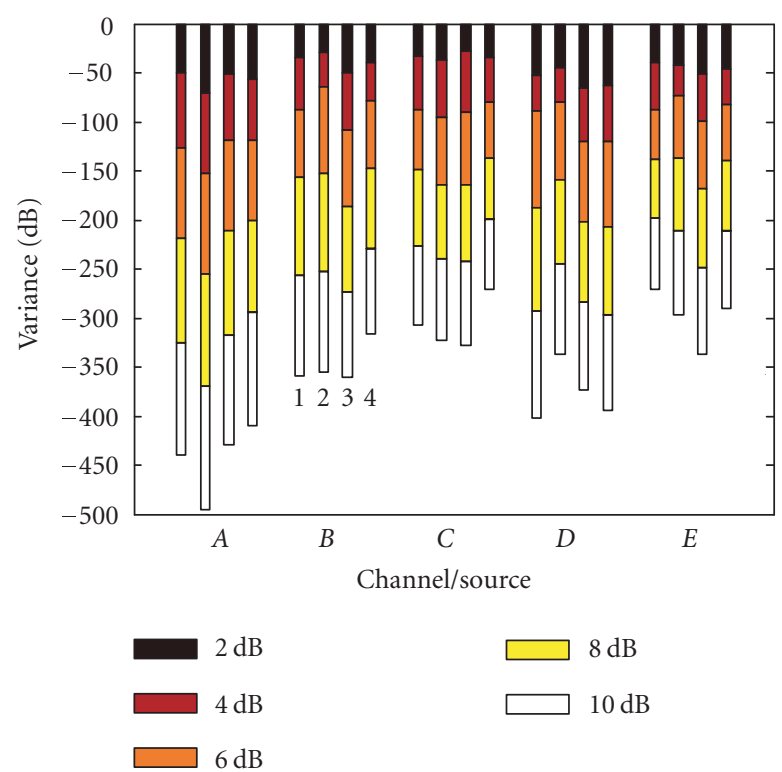

$\square 8 \mathrm{~dB}$

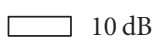

(b)

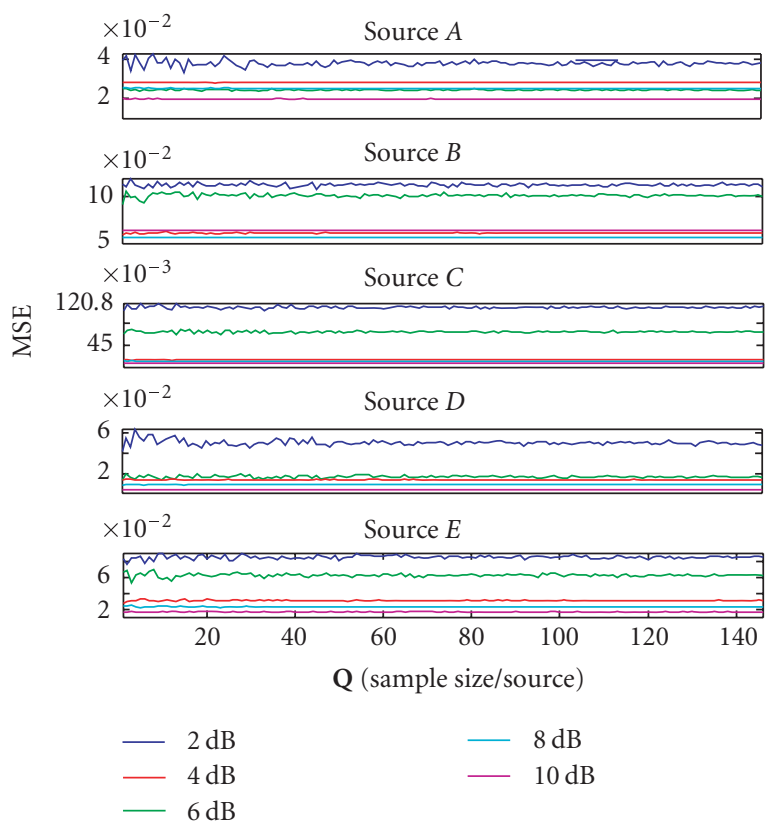

(d)

FIGURE 12: (a) Average estimates of $\mathbf{u}_{p}^{l}$ for the neural sources in Figure 11 (SNR $=4 \mathrm{~dB}$ ). (b) Variance of the estimates of $\mathbf{u}_{p}^{l}$ versus SNR. (c) Variance of the estimates of $\mathbf{u}_{p}^{l}$ versus sample size (of spikes) for each source, for each channel (SNR $=2 \mathrm{~dB}$ ). (d) MSE between the true $\mathbf{u}_{p}^{l}$ and the estimated $\mathbf{u}_{p}^{l}$ versus sample size versus SNR.

biophysical mechanism governing the concentration of the ion channels within a neural cell and membrane potentials $[15,44]$. Long-term nonstationarity has been observed as well due to cell migration, tissue relaxation, or electrode encapsulation $[38,43]$. Taken together, these temporal nonstationarities can be regarded as a fading process that may signficantly diminish the performance of any blind source separation algorithm. Spatial nonstationarity has to be taken into account as well especially when each neural source is treated as a distributed source $[34,36]$. This depends on the neural cell type [44], or when migration of the recording array relative to the neural population of interest occurs. However, temporal nonstationarity occurs at a faster rate compared to spatial nonstationarity. The case of spatial nonstationarity can be fully accounted for by making the mixing matrix time depedent. This case is outside the scope of this 
A

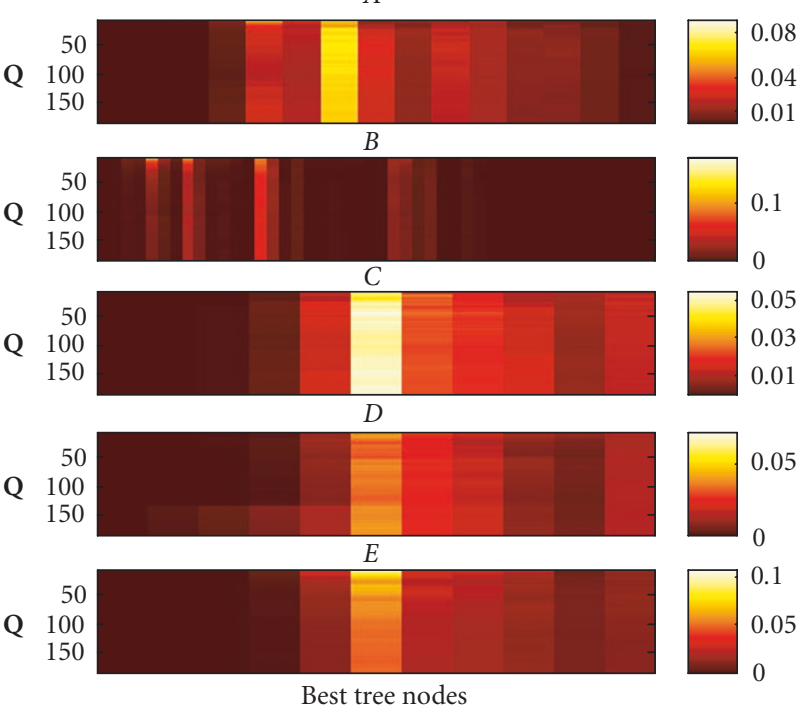

(a)

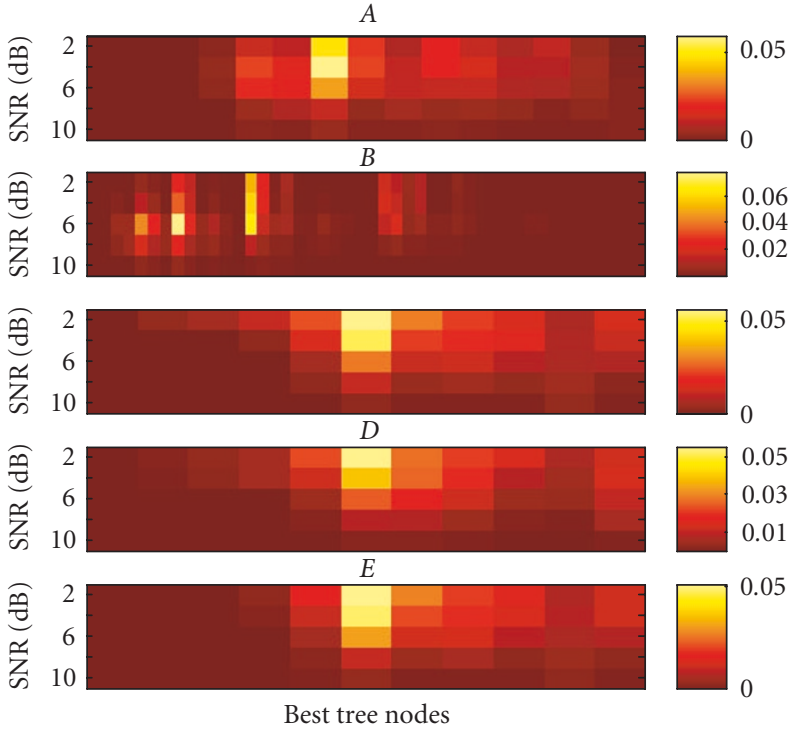

(b)

Figure 13: (a) Variance of $\lambda_{1}^{l}, l \in \mathfrak{J}_{p}(p=\{A, B, C, D, E\})$, versus sample size $\mathbf{Q}$ (SNR $=4 \mathrm{~dB}$ ) (b) Variance of $\lambda_{1}^{l}, l \in \mathfrak{J}_{p}$, versus SNR. (Nodes with zero eigenvalues are suppressed for clarity.)

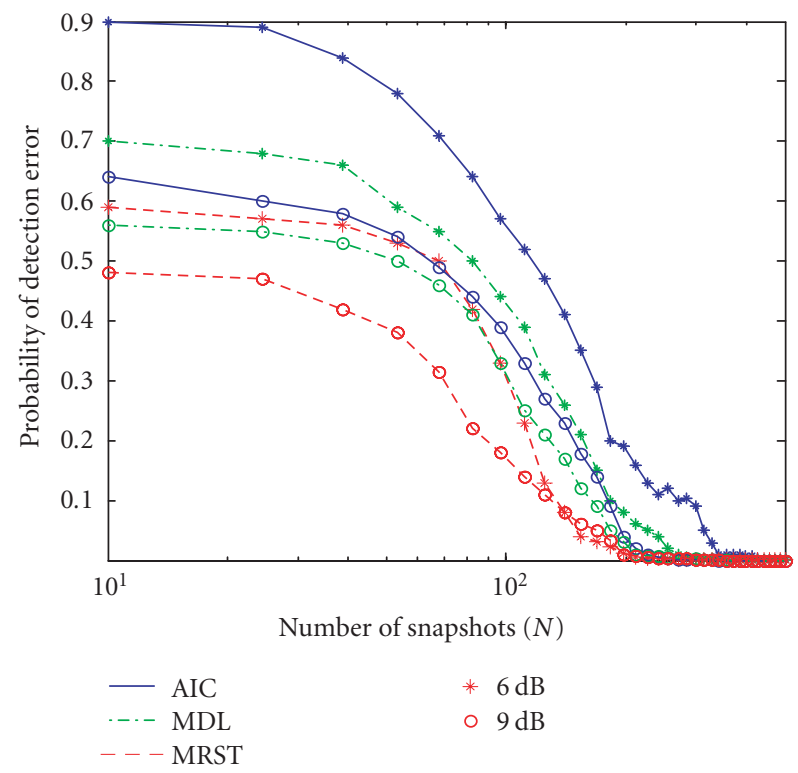

FIGURE 14: Probability of error versus the number of snapshots for the AIC, MDL, and multiresolution sphericity test in (14) [19].

paper. We will demonstrate that the algorithm is substantially robust to temporal variations.

The signals in Figure 15 represent a single source with 50 realizations over time in a burst activity. The cleaned version is illustrated for comparison. The multichannel signal is illustrated in (c) for event number 26. In (d) through (f), the features obtained are demonstrated. The set $\mathfrak{J}_{1}$ was invariant in all 50 realizations as well as the signal subspace as illustrated in (c) and (f), respectively. On the other hand, the eigenvalues $\lambda_{1}^{l}(k), 1 \leq k \leq 50$, relative to the eigenvalue of the first realization $\lambda_{1}^{l}(1)$ clearly demonstrate that the degradation in signal energy is efficiently captured by the change in the eigenvalues. Most notably, the gradual decrease in the eigenvalue in some nodes simultaneously occurs with a gradual increase in the eigenvalue in others. It is also clear that other nodes remain unchanged. These nodes can be interpreted as ones that are insensitive to energy degradation but rather capture other features in the waveform (e.g., zero crossing) more efficiently than others.

\section{CONCLUSION}

The problem of blind source separation of unknown correlated sources from noisy observations has been analyzed. We proposed a new solution to the problem that relies on exploiting the spatial diversity of the communication channel. In the context of blind source separation, our goal was to separate the correlated sources without having to estimate the unknown channel. Specifically, we showed that eigendecomposition of orthogonal transformations of the unknown signals is advantageous over classical time domain eigendecomposition when the orthogonality condition of signal sources cannot be met. The orthogonal transformation was carried out by means of the discrete wavelet transform and its overcomplete representation, the discrete wavelet packet transform, due of their excellent energy compaction ability. This property introduces large sparseness in the transform domain that constitutes a key element in reducing signal correlation thereby allowing the signal subspace to be reliably identified. We have further shown that the signal subspace remains invariant under temporal nonstationary conditions 


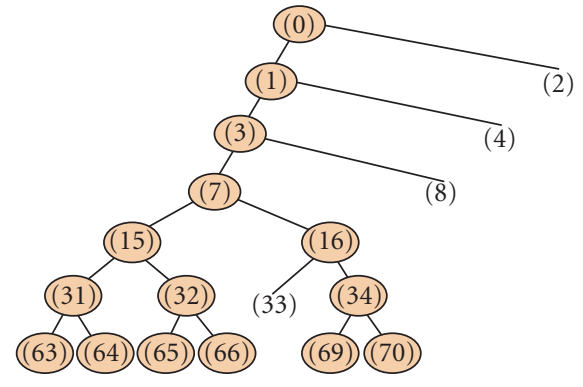

(a)
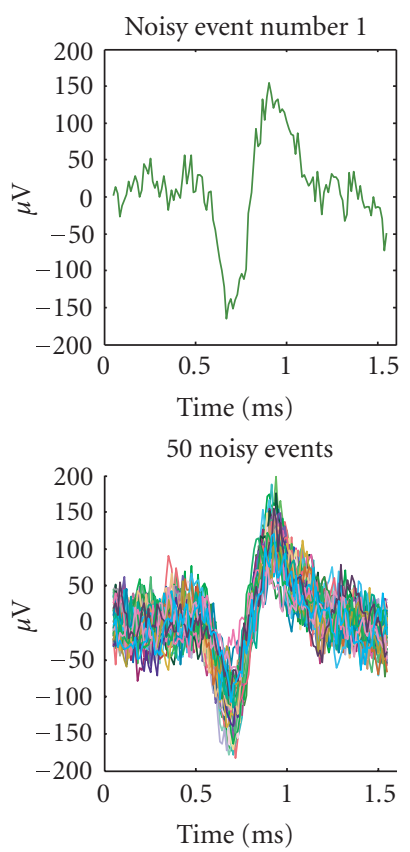

(b)

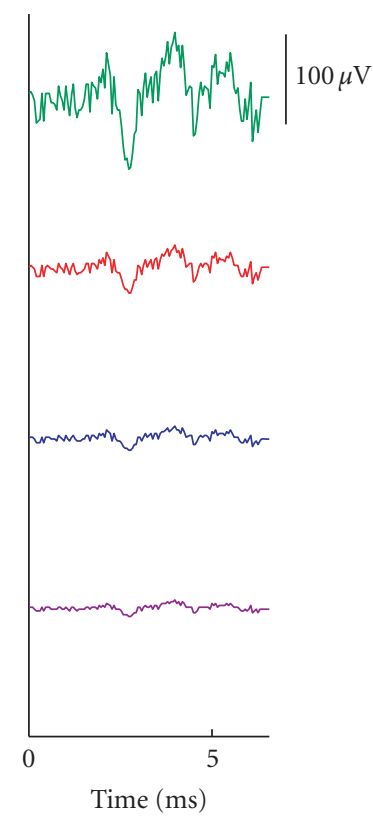

(c)

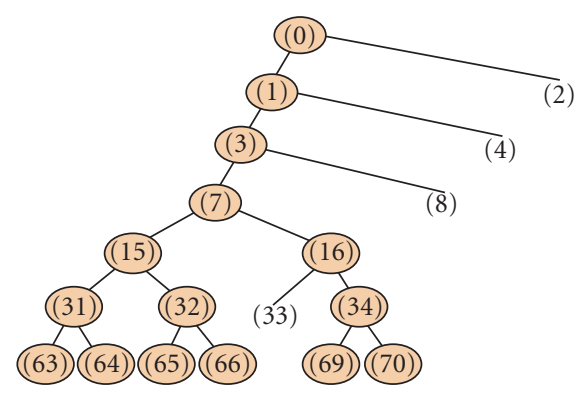

(d)

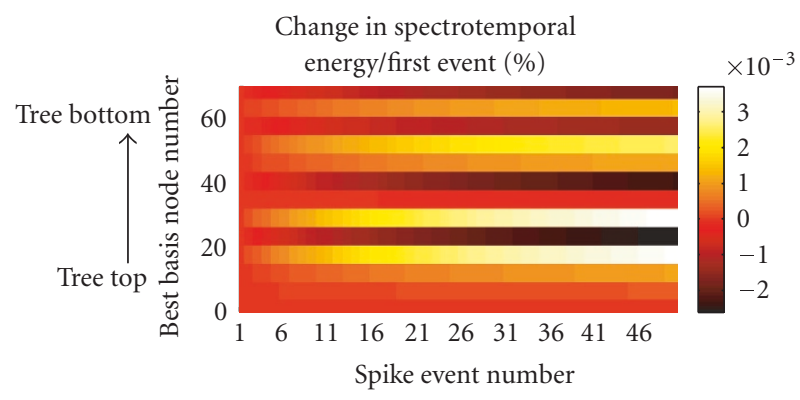

(e)

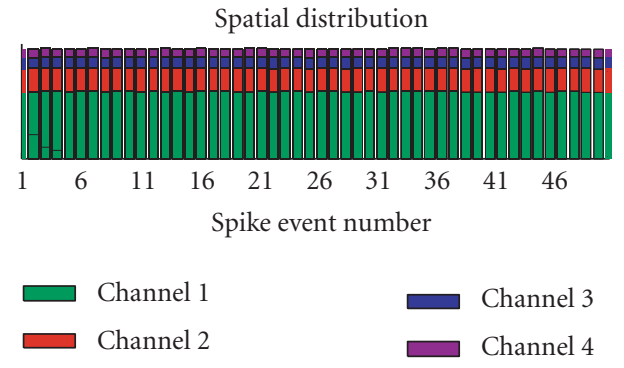

(f)

FIGURE 15: Tracking signal nonstationarity for an "attenuated-only" action potential of a bursting neuron. The largest amplitude spike is the first event. The events on channel 1 are superimposed in (a) and (b) for comparison. (c) Observed signal across a 4-channel array for event number 26. (d) Best basis tree set $\mathfrak{J}_{1}$ for each event. (e) The percentage change in eigenvalue $\lambda_{1}^{l}(k)$ relative to $\lambda_{1}^{l}(1)$ magnitude across the best basis set. The circled nodes are the best subbands were the spike waveform is represented, and the combination of positive and negative eigenvalues magnitudes changes in (e) is interpreted as a smooth migration of the spike projection from one node (where the change is negative) to other nodes (where the change is positive). In the later, the spike projection becomes stronger despite the loss of energy in the time domain waveform. 
of the signal, a property often observed in biological signals. We also described one possible implementation of the algorithm and showed its robustness to spatially and temporally correlated noise processes. In the context of classification, the approach utilizes a feature set that best describes the signal in space, time, and scale using three components, namely, the characteristic best basis binary tree set $\mathfrak{J}_{p}$, the eigenvalue distribution $\lambda_{p}^{l}, l \in \mathfrak{J}_{p}$, and the principal eigenvector $\mathbf{u}_{p}^{l}, l \in \mathfrak{J}_{p}$. This case was not treated in detail and is reported elsewhere [11].

Compared to MIMO systems, time and frequency diversity were not exploited in this approach because of the nature of the signal enviorment. It was assumed that the mixing is instantaneous and the search for signal structures was conducted across sensors. The particular case where noise is correlated with signals of interest is a major contribution of this approach, often simplified in similar applications. It is also obvious that the wavelet basis choice is of crucial importance to the separation process and may not be trivial in some cases since most wavelet bases share common properties like compact support while differing in other properties like symmetry and biorthogonality. The absence of knowledge about signal depedence complicates the problem to a large extent. For this paper, we chose from a dictionary of bases reported in the literature such as Haar, Daubechies, Symlet, Coiflet, and so forth. Within a chosen basis, different versions exist depending on the number of vanishing moments of the wavelet function. On the other hand, when the signal structure is unavailable, symlets of small order seemed to yield the best performance. We should note here that these results do not generalize for any sampling rate. The choice of another sampling rate may render another basis to perform better than symlets. One can argue that resampling the data to match a specific wavelet basis may be helpful and may lead to better results. When the signal is partially available in the form of signal templates, they can be used to design admissible wavelet basis to maximize a classification metric. Ideally, the wavelet basis should be selected to provide maximum separability between different sources. This topic is currently under investigation.

\section{REFERENCES}

[1] A. Hero, H. Messer, J. Goldberg, et al., "Highlights of statistical signal and array processing," IEEE Signal Processing Magazine, vol. 15 , no. 5 , pp. 21-64, 1998.

[2] G. Bienvenu and L. Kopp, "Optimality of high resolution array processing using the eigensystem approach," IEEE Transactions on Acoustics, Speech, and Signal Processing, vol. 31, no. 5, pp. 1235-1248, 1983.

[3] Y. Zhang, W. Mu, and M. G. Amin, "Subspace analysis of spatial time-frequency distribution matrices," IEEE Transactions on Signal Processing, vol. 49, no. 4, pp. 747-759, 2001.

[4] A.-J. Van Der Veen, E. F. Deprettere, and A. L. Swindlehurst, "Subspace-based signal analysis using singular value decomposition," Proceedings of the IEEE, vol. 81, no. 9, pp. 12771308, 1993.

[5] P. Stoica, O. Besson, and A. B. Gershman, "Direction-ofarrival estimation of an amplitude-distorted wavefront," IEEE Transactions on Signal Processing, vol. 49, no. 2, pp. 269-276, 2001.
[6] J. P. Le Cadre, "Parametric methods for spatial signal processing in the presence of unknown colored noise fields," IEEE Transactions on Acoustics, Speech, and Signal Processing, vol. 37, no. 7, pp. 965-983, 1989.

[7] P. Stoica, M. Viberg, and B. Ottersten, "Instrumental variable approach to array processing in spatially correlated noise fields," IEEE Transactions on Signal Processing, vol. 42, no. 1, pp. 121-133, 1994.

[8] H. Ye and R. D. DeGroat, "Maximum likelihood DOA estimation and asymptotic Cramer-Rao bounds for additive unknown colored noise," IEEE Transactions on Signal Processing, vol. 43, no. 4, pp. 938-949, 1995.

[9] F. Rieke, D. Warland, R. de Ruyter van Steveninck, and W. Bialek, Spikes: Exploring the Neural Code, MIT Press, Cambridge, Mass, USA, 3rd edition, 1997.

[10] K. G. Oweiss, "A systems approach for data compression and latency reduction in cortically controlled brain machine interfaces," IEEE Transactions on Biomedical Engineering, vol. 53, no. 7, pp. 1364-1377, 2006.

[11] K. G. Oweiss, "Integration of the temporal, spectral and spatial information for classifying multi-unit extracellular neural recordings," IEEE Transactions on Biomedical Engineering, in review

[12] K. G. Oweiss and D. J. Anderson, "A new technique for blind source separation using subband subspace analysis in correlated multichannel signal environments," in Proceedings of IEEE International Conference on Acoustics, Speech and Signal Processing (ICASSP '01), vol. 5, pp. 2813-2816, Salt Lake City, Utah, USA, May 2001.

[13] W. Hachem, F. Desbouvries, and P. Loubaton, "MIMO channel blind identification in the presence of spatially correlated noise," IEEE Transactions on Signal Processing, vol. 50, no. 3, pp. 651-661, 2002.

[14] D. Kotoulas, P. Koukoulas, and N. Kalouptsidis, "Subspace projection based blind channel order estimation of MIMO systems," IEEE Transactions on Signal Processing, vol. 54, no. 4, pp. 1351-1363, 2006.

[15] C. Gold, D. A. Henze, C. Koch, and G. Buzsáki, "On the origin of the extracellular action potential waveform: a modeling study," Journal of Neurophysiology, vol. 95, no. 5, pp. 31133128, 2006.

[16] D. Johnson and D. Dugeon, Array Signal Processing: Concepts and Techniques, Prentice Hall, Englewood Cliffs, NJ, USA, 1st edition, 1993.

[17] A. K. Jagannatham and B. D. Rao, "Whitening-rotation-based semi-blind MIMO channel estimation," IEEE Transactions on Signal Processing, vol. 54, no. 3, pp. 861-869, 2006.

[18] I. Daubechies, Ten Lectures on Wavelets, SIAM, Philadelphia, $\mathrm{Pa}, \mathrm{USA}, 1992$.

[19] K. G. Oweiss, "Source detection in correlated multichannel signal and noise fields," in Proceedings of IEEE International Conference on Acoustics, Speech and Signal Processing (ICASSP '03), vol. 5, pp. 257-260, Hong Kong, April 2003.

[20] R. R. Coifman and M. V. Wickerhauser, "Entropy-based algorithms for best basis selection," IEEE Transactions on Information Theory, vol. 38, no. 2, pt II, pp. 713-718, 1992.

[21] H. K. S. Mallat, D. Donoho, and A. S. Willsky, "Best basis algorithm for signal enhancement," in Proceedings of IEEE International Conference on Acoustics, Speech and Signal Processing (ICASSP '95), vol. 3, pp. 1561-1564, Detroit, Mich, USA, May 1995.

[22] J.-C. Pesquet, H. Krim, and H. Carfantan, "Time-invariant orthonormal wavelet representations," IEEE Transactions on Signal Processing, vol. 44, no. 8, pp. 1964-1970, 1996. 
[23] P. L. Dragotti and M. Vetterli, "Wavelet footprints: theory, algorithms, and applications," IEEE Transactions on Signal Processing, vol. 51, no. 5, pp. 1306-1323, 2003.

[24] M. S. Crouse, R. D. Nowak, and R. G. Baraniuk, "Waveletbased statistical signal processing using hidden Markov models," IEEE Transactions on Signal Processing, vol. 46, pp. 886902, 1998.

[25] D. L. Donoho, "De-noising by soft-thresholding," IEEE Transactions on Information Theory, vol. 41, no. 3, pp. 613-627, 1995.

[26] K. G. Oweiss and D. J. Anderson, "A new approach to array denoising," in Proceedings of the IEEE 34th Asilomar Conference on Signals, Systems and Computers (ASSC '00), vol. 2, pp. 1403-1407, Pacific Grove, Calif, USA, October-November 2000.

[27] D. B. Williams and D. H. Johnson, "Using the sphericity test for source detection with narrow-band passive arrays," IEEE Transactions on Acoustics, Speech, and Signal Processing, vol. 38, no. 11, pp. 2008-2014, 1990.

[28] T. Sugiyama, "On the distribution of the largest latent root of the covariance matrix," The Annals of Mathematical Statistics, vol. 38, no. 4, pp. 1148-1151, 1967.

[29] T. Sugiyama, "On the distribution of the latent vectors for principal component analysis," The Annals of Mathematical Statistics, vol. 36, no. 6, pp. 1875-1876, 1965.

[30] M. Vetterli and C. Herley, "Wavelets and filter banks: theory and design," IEEE Transactions on Signal Processing, vol. 40, no. 9, pp. 2207-2232, 1992.

[31] Y. Suhail and K. G. Oweiss, "A reduced complexity integer lifting wavelet-based module for real-time processing in implantable neural interface devices," in Proceedings of Annual International Conference of the IEEE Engineering in Medicine and Biology, vol. 2, pp. 4552-4555, San Francisco, Calif, USA, September 2004.

[32] I. Daubechies and W. Sweldens, "Factoring wavelet transforms into lifting steps," Journal of Fourier Analysis and Applications, vol. 4, no. 3, pp. 247-269, 1998.

[33] M.-W. Berry, "Large-scale sparse singular value computations," International Journal of Super-Computer Applications, vol. 6, no. 1, pp. 13-49, 1992.

[34] M. Nicolelis, Ed., Methods for Neural Ensemble Recordings, CRC Press, Boca Raton, Fla, USA, 1998.

[35] K. Wise, D. Anderson, J. Hetke, D. Kipke, and K. Najafi, "Wireless implantable microsystems: high-density electronic interfaces to the nervous system," Proceedings of the IEEE, vol. 92, no. 1, pp. 76-97, 2004.

[36] E. R. Kandel, J. H. Schwartz, and T. M. Jessell, Eds., Principles of Neural Science, chapter 2, Appleton \& Lange, Amsterdam, The Netherlands, 3rd edition, 1991.

[37] M. S. Lewicki, "A review of methods for spike sorting: the detection and classification of neural action potentials," Network: Computation in Neural Systems, vol. 9, no. 4, pp. 53-78, 1998.

[38] M. S. Fee, P. P. Mitra, and D. Kleinfeld, "Variability of extracellular spike waveforms of cortical neurons," Journal of Neurophysiology, vol. 76, no. 6, pp. 3823-3833, 1996.

[39] M. S. Fee, P. P. Mitra, and D. Kleinfeld, "Automatic sorting of multiple unit neuronal signals in the presence of anisotropic and non-Gaussian variability," Journal of Neuroscience Methods, vol. 69, no. 2, pp. 175-188, 1996.

[40] J. Rissanen, "Modeling by shortest data description," Automatica, vol. 14 , no. 5, pp. 465-471, 1978.

[41] W. Chen, K. M. Wong, and J. P. Reilly, "Detection of the number of signals: a predicted eigen-threshold approach," IEEE Transactions on Signal Processing, vol. 39, no. 5, pp. 1088-1098, 1991.
[42] Y. Wu and K.-W. Tam, "On determination of the number of signals in spatially correlated noise," IEEE Transactions on Signal Processing, vol. 46, no. 11, pp. 3023-3029, 1998.

[43] G. Buzsáki, "Large-scale recording of neuronal ensembles," Nature Neuroscience, vol. 7, no. 5, pp. 446-451, 2004.

[44] K. D. Harris, D. A. Henze, J. Csicsvari, H. Hirase, and G. Buzsáki, "Accuracy of tetrode spike separation as determined by simultaneous intracellular and extracellular measurements," Journal of Neurophysiology, vol. 84, no. 1, pp. 401$414,2000$.

Karim G. Oweiss obtained his B.S. (1993) and M.S. (1996) degrees in electrical engineering from the University of Alexandria, Egypt, and the Ph.D. degree in electrical engineering and computer science from the University of Michigan, Ann Arbor, in 2002. His Ph.D. dissertation exploited multiresolution analysis of multichannel neural recordings. He completed a postdoctoral training with the Biomedical Engineering

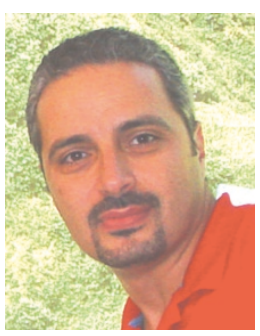
Department at the University of Michigan, Ann Arbor in the summer of 2002. In August 2002, he joined the Department of Electrical and Computer Engineering at Michigan State University, where he is currently an Assistant Professor and Director of the Neural Systems Engineering Laboratory. His research interests include statistical signal processing, information theory, data mining, multiresolution analysis, fast DSP algorithms with primary applications to neural signal processing, computational neuroscience, and brain machine interface technology. He is a Member of the IEEE, the Society for Neuroscience and the International Society for Optical Engineering. He is also a Member of the technical committee of the IEEE Signal Processing Society, the IEEE Circuits and Systems Society, and the IEEE Engineering in Medicine and Biology Society.

David J. Anderson received the B.S.E.E. degree from Rensselaer Polytechnic Institute, Troy, NY, and the M.S. and Ph.D. degrees from the University of Wisconsin, Madison. After completing a postdoctoral traineeship with the Laboratory of Neurophysiology, University of Wisconsin Medical School, he joined the University of Michigan, Ann Arbor, where he is now a Professor of electrical engineering and computer science, biomed-

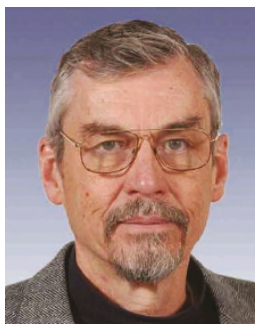
ical engineering, and otolaryngology. His research is in the areas of auditory physiology, neural recording device design, and signal processing of neural recordings. He is the founding Director of the University of Michigan Center for Neural Communication Technology, which conducts research on the development and use of silicon neural probe technology and distributes devices to neuroscientists worldwide. He is a Fellow of the American Institute for Medical and Biological Engineering, a Member of the Neuroscience Society, the Association for Research in Otolaryngology, and the Barany Society. 